Homology, Homotopy and Applications, vol. 8(1), 2006, pp.31-70

\title{
MODELLING FUNDAMENTAL 2-CATEGORIES FOR DIRECTED HOMOTOPY
}

\author{
MARCO GRANDIS
}

(communicated by George Janelidze)

\begin{abstract}
Directed Algebraic Topology is a recent field, deeply linked with ordinary and higher dimensional Category Theory. A 'directed space', e.g. an ordered topological space, has directed homotopies (which are generally non-reversible) and fundamental $n$-categories (replacing the fundamental $n$-groupoids of the classical case). Finding a simple model of the latter is a nontrivial problem, whose solution gives relevant information on the given 'space'; a problem which is also of interest in general Category Theory, as it requires equivalence relations which are more general than categorical equivalence. Taking on a previous work on 'The shape of a category up to directed homotopy', we study now the fundamental 2-category of a directed space. All the notions of 2-category theory used here are explicitly reviewed.
\end{abstract}

\section{Introduction}

Directed Algebraic Topology studies 'directed spaces' in some sense, where paths and homotopies cannot generally be reversed; for instance: ordered topological spaces, 'spaces with distinguished paths', 'inequilogical spaces', simplicial and cubical sets, etc. Its present applications deal mostly with the analysis of concurrent processes (see $[\mathbf{5}, \mathbf{6}, \mathbf{7}, \mathbf{8}, \mathbf{9}]$ ), but its natural range covers non-reversible phenomena, in any domain. Other references for Directed Algebraic Topology and its applications can be found in [14].

The study of invariance under directed homotopy is far richer and more complex than in the classical case, where homotopy equivalence between 'spaces' produces a plain equivalence of their fundamental groupoids, for which one can simply take - as a minimal model - the categorical skeleton. Our directed structures have, to begin with, a fundamental category $\uparrow \Pi_{1}(X)$. This must be studied up to appropriate notions of directed homotopy equivalence, which are more general than ordinary categorical equivalence: the latter would often be of no use, since the fundamental

This work was supported by MIUR Research Projects.

Received July 27, 2005; published on November 28, 2005.

2000 Mathematics Subject Classification: 18D05, 18A40, 55Pxx, 55Qxx, 55Uxx.

Key words and phrases: 2-categories, 2-functors, local adjunctions, homotopy theory, directed algebraic topology, fundamental 2-category.

Copyright (C) 2005, International Press. Permission to copy for private use granted. 
category of an ordered topological space, for instance, is always skeletal (the same situation shows that the fundamental monoids $\uparrow \pi_{1}\left(X, x_{0}\right)$, can be trivial, without $\uparrow \Pi_{1}(X)$ being so; cf. 1.2). Such a study has been carried out in a previous work [14], which will be cited as Part I; the references I.2 or I.2.3 refer, respectively, to its Section 2 or Subsection 2.3.

In Part I, we have introduced two (dual) directed notions, which take care, respectively, of variation 'in the future' or 'from the past': a future equivalence (a symmetric version of an adjunction, with two units) and its dual, a past equivalence (with two counits); and studied how to combine them. Minimal models of a category, up to these equivalences, have been introduced to better understand the 'shape' and properties of the category we are analysing, and of the process it represents. Part of this study is briefly recalled below, in Section 1. (The paper [6] has similar goals and results, based on different categorical tools, i.e. categories of fractions.)

As already noted in Part I, this analysis captures essential facts of many planar ordered spaces (subspaces of the ordered plane $\uparrow \mathbf{R}^{2}$ ), but may say little about objects embedded in the ordered space $\uparrow \mathbf{R}^{3}$, in the same way as the fundamental group does not recognise the singularity of a 2 -sphere. This is why we want to develop here a similar study of the 'shape' of 2-categories, adapted to study the fundamental 2-category $\uparrow \Pi_{2}(X)$ of an ordered space.

Outline. We begin with a brief review of the basic aspects of Part I (Section 1), ending with a motivation of a higher dimensional study (1.5). Lax natural transformations of 2-functors between 2-categories and the 'local adjunctions' they produce, introduced in the 1970s by Bunge [4], Gray [15] and Kelly [17], are recalled in Section 2 - and in the Appendix (Section 7) for more technical points. Sections 3 and 4 introduce and study future 2-equivalences between 2-categories, a symmetric version of a local adjunction. Theorem 3.4 shows that a future 2-equivalence has a canonical factorisation into two split future 2-equivalences (an analogous 1-dimensional property was proved in Part I), so that our 2-categories can be embedded into a common one, each of them as a future 2-retract (a sort of locally full, locally reflective subcategory); on the other hand, a future 2-retract and a past 2-retract of the same 2-category generate a global 2-dimensional model (4.2, 4.3). The definition of the fundamental 2-category of an ordered space is given in Section 5, and extended to more complex directed structures in 5.7; note that a map of preordered spaces induces a strict 2-functor between their fundamental 2-categories, but a homotopy produces a lax natural transformation (5.5). The previous notions are used in Section 6 to give a model of the fundamental 2-category of a 3-dimensional ordered space, the 'hollow cube', for which $\uparrow \Pi_{1}$ gives insufficient information (cf. 1.5).

\section{One-dimensional analysis of directed spaces}

We begin with a review of the basic ideas and results of Part I. A preorder relation is assumed to be reflexive and transitive; it is called a (partial) order if it is also anti-symmetric; using a preorder as the main notion has strong advantages, as recalled at the end of 1.1 . 


\subsection{Homotopy for preordered spaces}

The simplest topological setting where one can study directed paths and directed homotopies is likely the category pTop of preordered topological spaces and preorderpreserving continuous mappings; the latter will be simply called morphisms or maps, when it is understood we are in this category. (Richer settings will be recalled in Section 5.)

A (directed) path in the preordered space $X$ is a map $a: \uparrow[0,1] \rightarrow X$, defined on the standard directed interval $\uparrow \mathbf{I}=\uparrow[0,1]$ (with Euclidean topology and natural order). A (directed) homotopy $\varphi: f \rightarrow g: X \rightarrow Y$, from $f$ to $g$, is a map $\varphi: X \times \uparrow \mathbf{I} \rightarrow$ $Y$ coinciding with $f$ on the lower basis of the cylinder $X \times \uparrow \mathbf{I}$, with $g$ on the upper one. Of course, this (directed) cylinder is a product in pTop: it is equipped with the product topology and with the product preorder, where $(x, t) \prec\left(x^{\prime}, t^{\prime}\right)$ if $x \prec x^{\prime}$ in $X$ and $t \leqslant t^{\prime}$ in $\uparrow \mathbf{I}$.

The fundamental category $C=\uparrow \Pi_{1}(X)$ has, for arrows, the classes of directed paths up to the equivalence relation generated by directed homotopy with fixed endpoints; composition is given by the concatenation of consecutive paths.

Note that, generally, the fundamental category of a preordered space $X$ is not a preorder, i.e., it can have different arrows $x \rightarrow x^{\prime}$ between two given points (cf. 1.2); but any loop in $X$ lives in a zone of equivalent points and is reversible, so that all endomorphisms of $\uparrow \Pi_{1}(X)$ are invertible. Moreover, if $X$ is ordered, the fundamental category has no endomorphisms and no isomorphisms, except the identities, and is skeletal; therefore, ordinary equivalence of categories cannot yield any simpler model. Note also that, in this case, all the fundamental monoids $\uparrow \pi_{1}\left(X, x_{0}\right)=\uparrow \Pi_{1}(X)\left(x_{0}, x_{0}\right)$ are trivial. All these are crucial differences with the classical fundamental groupoid $\uparrow \Pi_{1}(X)$ of a space, for which a model up to homotopy invariance is given by the skeleton: a family of fundamental groups $\pi_{1}\left(X, x_{i}\right)$, obtained by choosing one point in each path-connected component of $X$.

The fundamental category of a preordered space can be computed by a van Kampen-type theorem, as proved in [11], Thm. 3.6, in a much more general setting ('d-spaces', cf. 5.7).

The forgetful functor $U:$ p Top $\rightarrow$ Top with values in the category of topological spaces has both a left and a right adjoint, $D \dashv U \dashv C$, where $D X$ (resp. $C X$ ) is the space $X$ with the discrete order (resp. the coarse preorder). Therefore, $U$ preserves limits and colimits. The standard embedding of Top in pTop will be the coarse one, so that all (ordinary) paths in $X$ are directed in $C X$, and we can view ordinary Algebraic Topology as embedded into the Directed one. Note that the category of ordered spaces does not allow for such an embedding, and has different colimits.

\subsection{The fundamental category of a square annulus}

An elementary example will give some idea of the analysis developed in Part I. Let us start from the standard ordered square $\uparrow[0,1]^{2}$, with the Euclidean topology and the product order

$$
(x, y) \leqslant\left(x^{\prime}, y^{\prime}\right) \quad \text { if : } \quad x \leqslant x^{\prime}, y \leqslant y^{\prime},
$$


and consider the (compact) ordered subspace $A$ obtained by taking out the open square $] 1 / 3,2 / 3{ }^{2}$ (marked with a cross), a sort of 'square annulus'

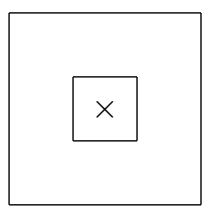

$A$

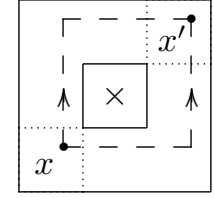

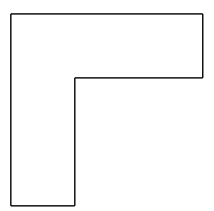

$L$

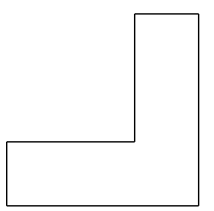

$L^{\prime}$

Its directed paths are, by definition, the continuous order-preserving maps $\uparrow[0,1] \rightarrow A$ defined on the standard ordered interval, and move 'rightward and upward' (in the weak sense). Directed homotopies of such paths are continuous order-preserving maps $\uparrow[0,1]^{2} \rightarrow A$. The fundamental category $C=\uparrow \Pi_{1}(A)$ has, for arrows, the classes of directed paths up to the equivalence relation generated by directed homotopy (with fixed endpoints, of course).

In our example, the fundamental category $C$ has some arrow $x \rightarrow x^{\prime}$ provided that $x \leqslant x^{\prime}$ and both points are in $L$ or $L^{\prime}$ (the closed subspaces represented above). Precisely, there are two arrows when $x \leqslant p=(1 / 3,1 / 3)$ and $x^{\prime} \geqslant q=(2 / 3,2 / 3)$ (as in the second figure above), and one otherwise. This evident fact can be easily proved with the 'van Kampen' theorem recalled above, using the subspaces $L, L^{\prime}$ (whose fundamental category is the induced order).

Thus, the whole category $C$ is easy to visualise and 'essentially represented' by the full subcategory $E$ on four vertices $0, p, q, 1$ (the central cell does not commute)
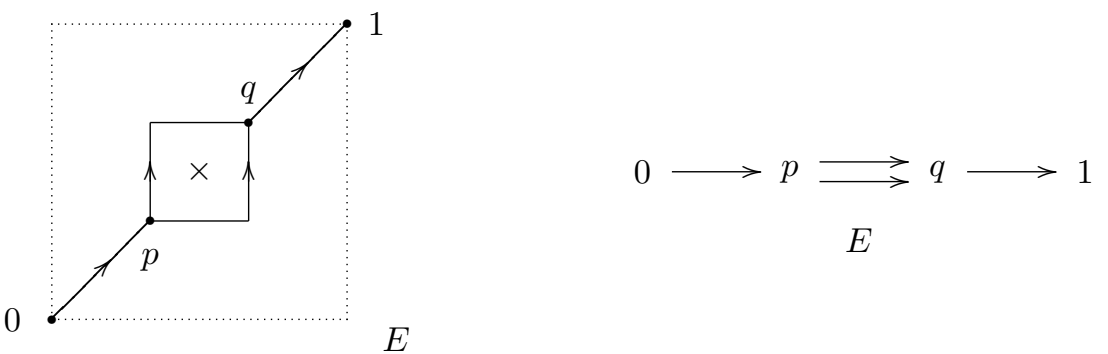

But $E$ is far from being equivalent to $C$, as a category, since $C$ is already a skeleton, in the ordinary sense. The situation can be analysed as follows, in $E$ :

- the action begins at 0 , from where we move to the point $p$,

- $p$ is an (effective) future branching point, where we have to choose between two paths,

- which join at $q$, an (effective) past branching point,

- from where we can only move to 1 , where the process ends. 
(Definitions and properties of regular and branching points can be found in I.6).

In order to make precise how $E$ can 'model' the category $C$, we proved in Part I (and will recall below) that $E$ is both future equivalent and past equivalent to $C$, and actually is the 'join' of a minimal 'future model' with a minimal 'past model' of the latter.

\subsection{Future equivalence of categories}

A future equivalence $(f, g ; \varphi, \psi)(\mathrm{I} .2 .1)$ between the categories $C, D$ is a symmetric version of an adjunction, with two units. It consists of a pair of functors and a pair of natural transformations (i.e., directed homotopies), the units, satisfying two coherence conditions:

$$
\begin{gathered}
f: C \rightleftarrows D: g, \quad \varphi: 1_{C} \rightarrow g f, \quad \psi: 1_{D} \rightarrow f g, \\
f \varphi=\psi f: f \rightarrow f g f, \quad \varphi g=g \psi: g \rightarrow g f g \quad \text { (coherence). }
\end{gathered}
$$

Note that the directed homotopies $\varphi, \psi$ proceed from the identities to the composites $g f, f g$ ('in the future'). Dually, past equivalences have counits, in the opposite direction (with the composed functors 'in the past').

Future equivalences compose (in the same way as adjunctions), and yield an equivalence relation of categories. A property (making sense in a category, or for a category) is said to be future invariant if it is preserved by future equivalences.

An adjunction $f \dashv g$ with invertible counit $\varepsilon: f g \cong 1$ amounts to a future equivalence with invertible $\psi=\varepsilon^{-1}$. In this case, a 'split' future equivalence, $D$ can be identified with a full reflective subcategory of $C$ (also called a future retract, I.2.4). But, in a general future equivalence, $f$ need not determine $g$.

Theorem I.2.5 shows that two categories are future equivalent if and only if they can be embedded into a common one, as full reflective subcategories. Dually, past equivalence is the equivalence relation generated by being a full coreflective subcategory.

\subsection{Minimal one-dimensional models}

In our example (1.2), the category $C=\uparrow \Pi_{1}(A)$ has a least full reflective subcategory $F$, which is future equivalent to $C$ and minimal as such; its objects are a future branching point $p$ (where we must choose between different ways out of it) and a maximal point 1 (where one cannot further proceed); they form the future spectrum $\mathrm{sp}^{+}(C)$ (as defined in I.7.2)
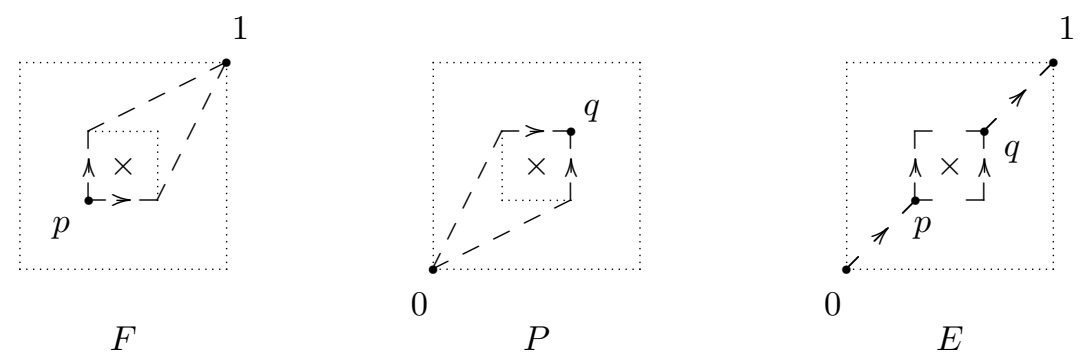
Dually, we have the least full coreflective subcategory $P$, whose objects form the past spectrum $s p^{-}(C)=\{0, q\}$.

Putting together the information coming from a past and a future spectrum, the pf-spectrum $E=S p(C)$ is the full subcategory of $C$ on the set of objects $s p(C)=s p^{-}(C) \cup s p^{+}(C)$ (I.7.6). It is linked to $C$ by a diagram formed of four commutative squares

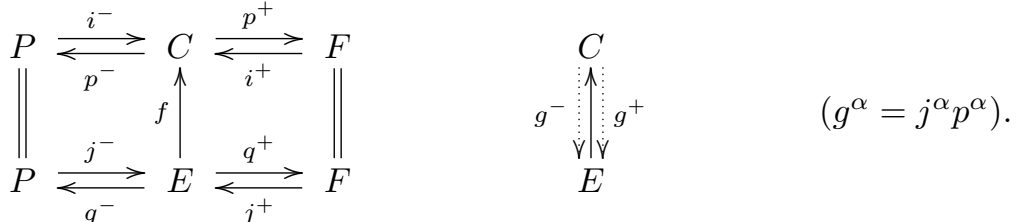

Adding the two functors $g^{\alpha}=j^{\alpha} p^{\alpha}: C \rightarrow E$ (where $\alpha= \pm$ ), $E$ becomes a minimal injective model of the category $C$, in a precise sense, which we recall now (all this is not technically required for the sequel, but will suggest how to proceed for dimension 2, in Section 4).

First, a category $E$ is made an injective model of $C$ (I.4.1) by assigning a pf-injection, or pf-embedding, $E \leftrightarrows C$. This consists of a full embedding $f: E \rightarrow C$ (full, faithful and injective on objects) which belongs at the same time to a past equivalence $\left(f, g^{-} ; \varepsilon_{E}, \varepsilon_{C}\right)$ and a future equivalence $\left(f, g^{+} ; \eta_{E}, \eta_{C}\right)$

$$
\begin{aligned}
& f: E \leftrightarrows C: g^{-}, g^{+} \\
& \varepsilon_{E}: g^{-} f \rightarrow 1_{E}, \quad \varepsilon_{C}: f g^{-} \rightarrow 1_{C}, \\
& f \varepsilon_{E}=\varepsilon_{C} f: f g^{-} f \rightarrow f, \quad \varepsilon_{E} g^{-}=g^{-} \varepsilon_{C}: g^{-} f g^{-} \rightarrow g^{-} \text {, } \\
& \eta_{E}: 1_{E} \rightarrow g^{+} f, \quad \eta_{C}: 1_{C} \rightarrow f g^{+}, \\
& f \eta_{E}=\eta_{C} f: f \rightarrow f g^{+} f, \quad \eta_{E} g^{+}=g^{+} \eta_{C}: g \rightarrow g^{+} f g^{+} .
\end{aligned}
$$

(A coherence property between these two structures automatically holds, I.3.3. By I.3.4, it suffices to assign the three functors $f, g^{-}, g^{+}$- the first being a full embedding - together with the natural transformations $\varepsilon_{C}$ and $\eta_{C}$, under the conditions $f g^{-} \varepsilon_{C}=\varepsilon_{C} f g^{-}, f g^{+} \eta_{C}=\eta_{C} f g^{+}$.)

Secondly, we say that $E$ is a minimal injective model of $C$ (I.5.2) if:

(i) $E$ is an injective model of every injective model $E^{\prime}$ of $C$,

(ii) every injective model $E^{\prime}$ of $E$ is isomorphic to $E$.

We also say that $E$ is a strongly minimal injective model if it satisfies the stronger condition (i'), together with (ii):

(i') $E$ is an injective model of every category injectively equivalent to $C$,

where two categories are said to be injectively equivalent if they can be linked by a finite chain of pf-embeddings, forward or backward (I.4.1).

Finally, Theorems I.8.4 and I.8.6 prove that, if a category $C$ has a pf-spectrum (defined in I.7.2), this is a strongly minimal injective model of $C$, determined up to a unique isomorphism. (More generally, the minimal injective model of a category, when existing, is determined up to isomorphism but the isomorphism itself need not be determined; cf. I.5.5, I.5.6). 


\subsection{The hollow cube}

The analysis recalled above, based on the fundamental category, gives relevant information for planar ordered spaces (subspaces of $\uparrow \mathbf{R}^{2}$ ), also in much more complicated examples (see I.9). But it may be insufficient for higher dimensional singularities.

The simplest case (already considered in I.9.8) is a 3-dimensional analogue of our previous example, the 'hollow cube' $X \subset \uparrow[0,1]^{3}$ represented below (again, an ordered compact space):

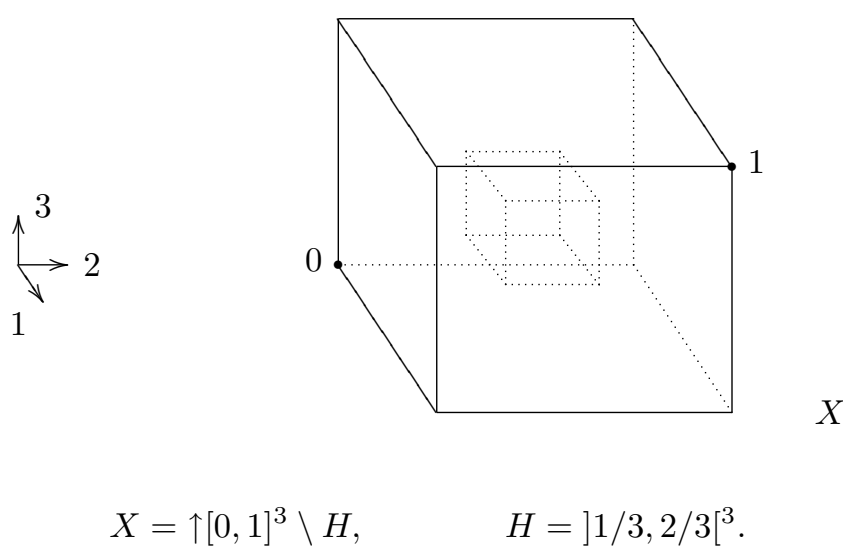

The fundamental category $C=\uparrow \Pi_{1}(X)$ seems to say little about this space: $C$ has an initial object 0 and a terminal object 1 , whence it is future contractible (to its object 1 ) and past contractible as well (to 0 ); its minimal injective model is the category $\mathbf{2}=\{0 \rightarrow 1\}$ (cf. I.5.4).

Now, as already remarked in Part I, this injective model is not faithful: the original category $C$ is not a preorder, since $C(x, y)$ has two arrows when $x, y$ are suitably placed 'around' the obstruction (precisely because we are working within directed homotopy theory). One might therefore try to extract a better information from $C$, using faithful models. However, we are not able to find any simple one (and likely, there is no finite one).

Here, we shall study the fundamental 2-category $C_{2}=\uparrow \Pi_{2}(X)$, trying to reproduce one dimension up the previous study of $\uparrow \Pi_{1}(A)$, for the 'square annulus' (1.4). This will be done in Section 6, after preparing the new tools.

\section{Lax natural transformations and local adjunctions}

We review now the main tools of 2-dimensional category theory which will be used in this paper: (strict) 2-functors, their lax natural transformations, their modifications and local adjunctions as introduced in $[4,15,17]$. We shall mostly follow Bunge's terminology [4], slightly adapted (cf. 2.2). 


\subsection{Notation}

Dealing with (strict) 2-categories, there is some advantage in beginning with the more general notion of sesquicategory, where we only have a vertical composition of cells and a horizontal 'whisker' composition of cells with arrows [19].

We shall use the following notation. In a sesquicategory $X$ we have objects $x, y, \ldots$, maps $a: x \rightarrow y, \ldots$ and cells $\alpha: a \rightarrow b: x \rightarrow y, \ldots$ Maps have an associative composition, written $b a($ or $b \cdot a)$, with identities $1_{x}$. Cells have a main composition $\beta \alpha: a \rightarrow c: x \rightarrow y$ (also written $\beta \cdot \alpha$ ), as in the left diagram below, which is associative and has identities $1_{a}$ (in the sequel, the terms 'horizontal' and 'vertical' will only be used for the pastings of the associated double cells; see below)

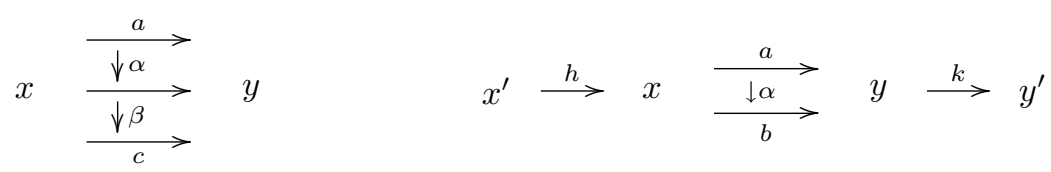

Cells and maps have a whisker composition $k \alpha h: k a h \rightarrow k b h: x^{\prime} \rightarrow y^{\prime}$ (as in the right diagram above) such that:

$$
\begin{array}{ll}
1_{y} \alpha 1_{x}=\alpha, & k^{\prime}(k \alpha h) h^{\prime}=\left(k^{\prime} k\right) \alpha\left(h h^{\prime}\right), \\
k 1_{a} h=1_{k a h}, & k(\beta \alpha) h=(k \beta h)(k \alpha h) .
\end{array}
$$

This sesquicategory 'is' a 2-category if the 'reduced interchange axiom' holds:

$$
x \underset{b}{\stackrel{\frac{a}{\downarrow}}{\longrightarrow}} y \underset{d \gamma}{\stackrel{\frac{c}{\longrightarrow}}{\longrightarrow}} z \quad \quad \gamma b \cdot c \alpha=d \alpha \cdot \gamma a,
$$

in which case, one can define the lower composition $\gamma \circ \alpha: c a \rightarrow d b: x \rightarrow z$ as the previous common result. We shall generally work with 2-categories (mostly without using the lower composition of cells).

A (strict) 2-functor $f: X \rightarrow Y$ takes items of $X$ to similar items of $Y$, preserving identities and compositions. We shall only use such strict morphisms.

It will be useful to use pasting. This amounts to identifying a 2-category with its strict double category of quintets (due to C. Ehresmann), with double cells as in the left diagram below (provided by a 2 -cell $\varphi: v a \rightarrow b u$ )
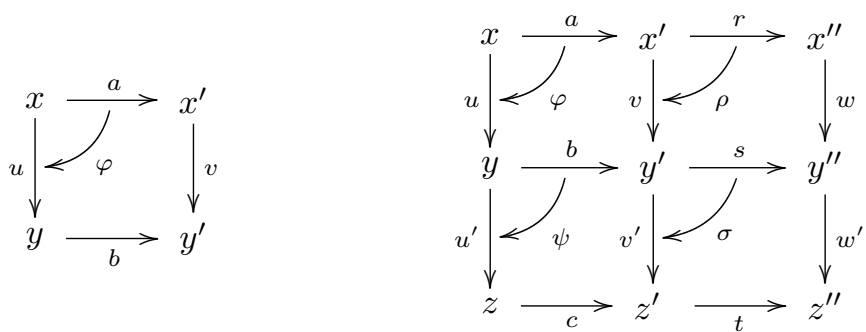

The horizontal and vertical compositions of double cells are obtained in the obvious way, from the vertical composition of 2-cells and the whisker composition 
of 2-cells with arrows, as in the right diagram above

$$
\begin{aligned}
& \varphi \otimes_{h} \rho=s \varphi \cdot \rho a: w r a \longrightarrow s v a \longrightarrow s b u, \\
& \varphi \otimes_{v} \psi=\psi u \cdot v^{\prime} \varphi: v^{\prime} v a \longrightarrow v^{\prime} b u \longrightarrow c u^{\prime} u .
\end{aligned}
$$

Note that, more generally, these horizontal and vertical compositions of double cells can be defined (and are associative) for a sesquicategory $X$; then, $X$ is a 2-category (satisfies the reduced interchange axiom) if and only if its double cells form a double category (i.e., the horizontal and vertical pastings satisfy the middlefour interchange axiom).

\subsection{Lax natural transformations}

A lax natural transformation $\varphi: f \rightarrow g: X \rightarrow Y$ (between strict 2-functors) assigns:

(i) to every object $x$ in $X$, a map $\varphi x: f x \rightarrow g x$ in $Y$,

(ii) to every map $a: x \rightarrow x^{\prime}$ in $X$, a comparison cell $\varphi a: \varphi x^{\prime} \cdot f a \rightarrow g a \cdot \varphi x$ in $Y$,

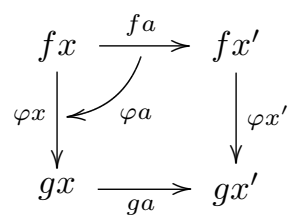

so that the following axioms hold:

(lnt.1) if $a=1_{x}$, then $\varphi a=1: \varphi x \rightarrow \varphi x$,

(lnt.2) if $c=b a: x \rightarrow x^{\prime} \rightarrow x^{\prime \prime}$, then $\varphi a \otimes_{h} \varphi b=\varphi c$ (i.e., $\varphi c=(g b \cdot \varphi a)(\varphi b \cdot f a)$ ),

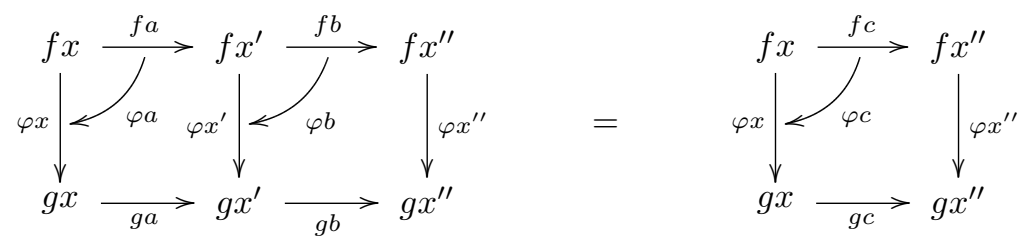

(lnt.3) given a cell $\alpha: a \rightarrow b: x \rightarrow x^{\prime}$ in $X$, then $f \alpha \otimes_{v} \varphi b=\varphi a \otimes_{v} g \alpha$ (i.e., $\left.\varphi b\left(\varphi x^{\prime} \cdot f \alpha\right)=(g \alpha \cdot \varphi x) \varphi a\right)$

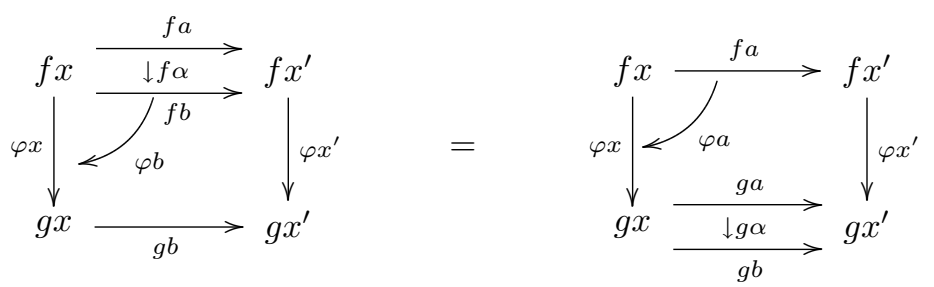

The cell-dual notion, an op-lax natural transformation, has comparison cells in the opposite direction: $g a \cdot \varphi x \rightarrow \varphi x^{\prime} \cdot f a$. 
The present choice of the leading form, with cells $\varphi a: \varphi x^{\prime} \cdot f a \rightarrow g a \cdot \varphi x$ directed from $f$ to $g$, agrees with Bunge's paper [4], but differs from Gray's [15] (where 'd' stands for down) and Kelly's [17]:

lax natural transformation: op-lax natural transformation:

[15]: quasi ${ }_{\mathrm{d}}$-natural transformation, quasi-natural transformation,

[17]: op-lax natural transformation, lax natural transformation.

We follow Bunge's choice because it is well-related with 'local adjunctions' (2.4; see the remark at the end of 2.5).

\subsection{Modifications}

A modification $M: \varphi \rightarrow \psi: f \rightarrow g: X \rightarrow Y$ (between lax natural transformations of 2-functors) assigns to every object $x$ in $X$ a cell $M x: \varphi x \rightarrow \psi x: f x \rightarrow g x$ in $Y$, so that the following axiom holds:

(mdf) if $a: x \rightarrow x^{\prime}$, then:

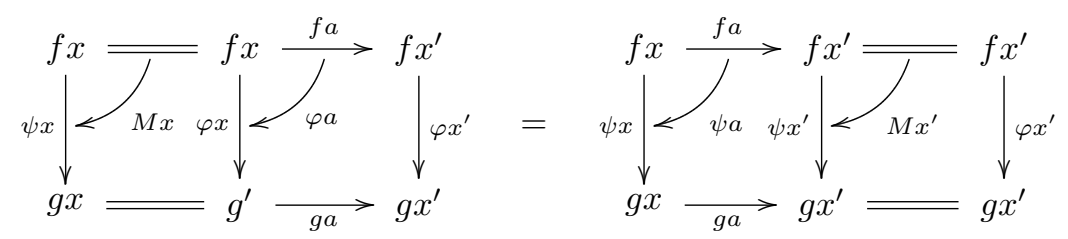

The calculus of lax natural transformations and modifications, under their compositions, is deferred to the Appendix, Section 7.

\subsection{Local adjunctions}

A local adjunction $(f, g ; \eta, \varepsilon ; L, R)$ between the 2-categories $X, Y$ consists of a pair of 2-functors, a pair of lax natural transformations and a pair of modifications (replacing the triangular identities)

$$
f: X \rightleftarrows Y: g,
$$

$$
\begin{array}{lr}
\eta: 1_{X} \rightarrow g f: X \rightarrow X & \text { (unit), } \\
\varepsilon: f g \rightarrow 1_{Y}: Y \rightarrow Y & \text { (counit), } \\
L: \varepsilon f \cdot f \eta \rightarrow 1_{f}: f \rightarrow f: X \rightarrow Y & \text { (left triangular comparison), } \\
R: 1 g \rightarrow g \varepsilon \cdot \eta g: g \rightarrow g: Y \rightarrow X & \text { (right triangular comparison). }
\end{array}
$$

This will be called a coherent local adjunction if it satisfies the coherence axioms:

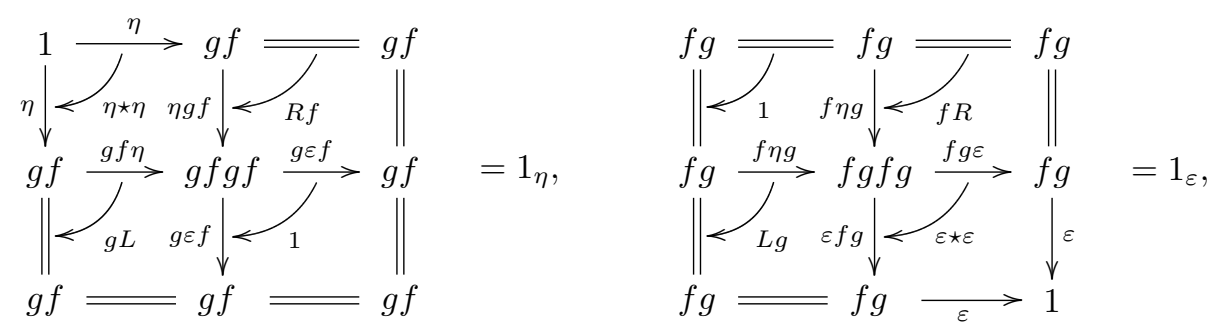


with obvious modifications $\eta \star \eta, \varepsilon \star \varepsilon$ (graded composition of lax natural transformations, 7.3). In a strictly coherent local adjunction the triangular comparisons are identities $(L=1, R=1)$ and the coherence axioms reduce to $\eta \star \eta=1, \varepsilon \star \varepsilon=1$.

The cell-dual notion, with op-lax natural transformations $\eta: 1_{X} \rightarrow g f, \varepsilon: f g \rightarrow$ $1_{Y}$ and comparisons $L: 1_{f} \rightarrow \varepsilon f \cdot f \eta, R: g \varepsilon \cdot \eta g \rightarrow 1_{g}$, will be called an op-local adjunction.

A coherent local adjunction (in the present sense) is called a 'formal lax adjunction' in [4], 3.1 (where $f$ need not be strict). An op-local adjunction is called a 'weak quasi-adjunction' in [15], I.7.1, and a 'quasi-adjunction' when coherence holds. The term 'local adjunction', motivated below, appeared in $[\mathbf{2}, \mathbf{3}, \mathbf{1 6}]$, with similar meanings.

Local adjunctions are closed under composition ([15], I.7.3).

\subsection{The local behaviour}

The name of local adjunction is motivated by the fact that this structure is linked with ordinary adjunctions at the 'local' level, of hom-categories.

More precisely, a local adjunction $f \dashv g$ (18) induces, for every pair of objects $x$ (in $X$ ) and $y$ (in $Y$ ), a sort of 'pre-adjunction' $f^{\prime} \dashv g^{\prime}$ (without triangular identities), between ordinary functors naturally associated with $f$ and $g$, respectively:

$$
\begin{aligned}
& f^{\prime}: X(x, g y) \rightleftarrows Y(f x, y): g^{\prime}, \\
& f^{\prime}(a: x \rightarrow g y)=\varepsilon y \cdot f a: f x \rightarrow y, \quad g^{\prime}(b: f x \rightarrow y)=g b \cdot \eta x: x \rightarrow g y, \\
& \eta^{\prime}: 1 \rightarrow g^{\prime} f^{\prime}: X(x, g y) \rightarrow X(x, g y), \quad \varepsilon^{\prime}: f^{\prime} g^{\prime} \rightarrow 1: Y(f x, y) \rightarrow Y(f x, y) \text {. }
\end{aligned}
$$

The components $\eta^{\prime} a$ and $\varepsilon^{\prime} b$ are cells, defined by the following pastings
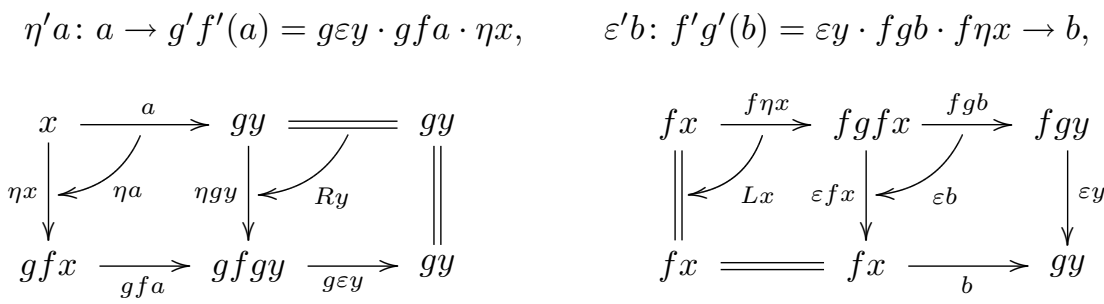

If the original local adjunction is coherent, then (20) is an adjunction (satisfies the triangular identities). On the other hand, a coherent op-local adjunction $f \dashv g$ produces an adjunction $g^{\prime} \dashv f^{\prime}$ whose 'direction' does not agree with the given one.

\subsection{Local terminal objects}

Local adjunctions produce local limits and colimits, as studied in the references cited above. In a very elementary way, let us consider the 2-category $X=\operatorname{Rel} \mathbf{A b}$ of (additive) relations of abelian groups and the trivial 2-category $\mathbf{1}$ on one object $\star$, linked by the following 2 -functors forming a retraction ( 0 denotes the null group)

$$
p: X \rightleftarrows 1: i, \quad i(\star)=0 \quad(p i=1) .
$$

Adding units or counits, we can get various (op)local adjunctions (but no 2-adjunction, since $X$ has neither a terminal nor an initial object). 
(a) First, we have a strictly coherent local adjunction $p \dashv i$, with trivial counit $p i=1$ and unit $\eta: 1_{X} \rightarrow i p$, sending an object $A$ to the greatest relation $\eta A: A \rightarrow 0$ (with graph $A \times 0$ ). The following diagram shows the comparison cell of $\eta$, on an arbitrary relation $a: A \rightarrow B$

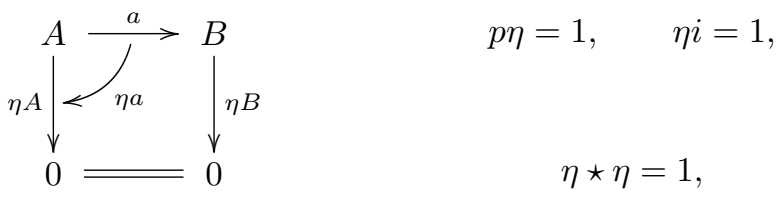

while the coherence properties ((lnt.1-3) in 2.2) are automatically satisfied, because a 2 -category of relations is locally ordered: its cells are determined by their domain and codomain.

We can say that this adjunction presents the null group 0 as a local terminal object of $X$.

(b) Secondly, we have a coherent local adjunction $i \dashv p$ with trivial unit $1=p i$ and counit $\varepsilon: i p \rightarrow 1_{X}$, sending an object $A$ to the least relation $\varepsilon A: 0 \rightarrow A$ (with graph $0 \times 0)$

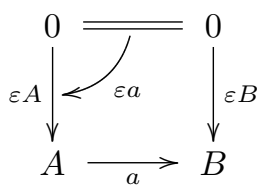

$$
\begin{gathered}
p \varepsilon=1, \quad \varepsilon i=1, \\
\varepsilon \star \varepsilon=1 .
\end{gathered}
$$

This presents the null group 0 as a local initial object, in $X$.

(c) But we also have two coherent op-local adjunctions $p \dashv i$ and $i \dashv p$.

The unit of the first is the op-lax natural transformation $\eta^{\prime}: 1_{X} \rightarrow i p$ where $\eta^{\prime} A=(\varepsilon A)^{\sharp}: A \rightarrow 0$ is the least relation (see the left diagram below)
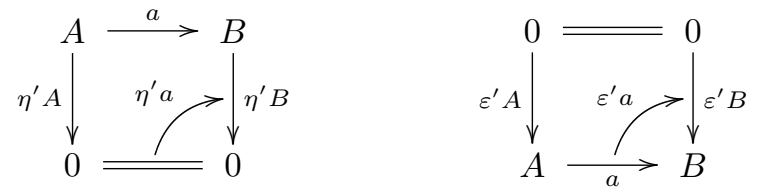

while the counit of second is the op-lax natural transformation $\varepsilon^{\prime}: i p \rightarrow 1_{X}$, $\varepsilon^{\prime} A=(\eta A)^{\sharp}$. These adjunctions present 0 as an op-local terminal and op-local initial object, respectively.

The points (a) and (b) seem also to show that lax natural transformations, in the present sense, play a leading role with respect to the dual notion: in fact, the presentation of 0 as a local terminal object comes with terminal relations $A \rightarrow 0$ (terminal 'objects' in the order-category Rel $\mathbf{A b}(A, 0)$ ), and the presentation as a local initial object comes with initial relations $0 \rightarrow A$.

For relations of sets, a similar argument would show that the 2-functor $p$ : Rel Set $\rightarrow 1$ has two (non-isomorphic) strictly coherent local right adjoints, corresponding to the empty set and the singleton. But now, the transformations pertaining to the empty set (which is 2-terminal and 2-initial) are 2-natural. 
Homology, Homotopy and Applications, vol. 8(1), 2006

\section{Future and past 2-equivalences}

As in the 1-dimensional case, directed homotopy equivalence of 2-categories comes in two dual forms, which identify future invariants or past invariants, respectively.

\subsection{Future 2-equivalences}

We shall work with strict 2-functors, their lax natural transformations and modifications, as recalled in the previous section (or in Section 7, for their compositions).

A future 2-equivalence $(f, g ; \varphi, \psi ; F, G)$ between the 2-categories $X, Y$ will consist of a pair of 2-functors, a pair of lax natural transformations (2.2), the units, and a pair of modifications (2.3), the comparisons

$$
\begin{aligned}
& \qquad: X \rightleftarrows Y: g \\
& \varphi: 1_{X} \rightarrow g f: X \rightarrow X, \\
& F: \psi f \rightarrow f \varphi: f \rightarrow f g f: X \rightarrow Y, \\
& G: \varphi g \rightarrow g \psi: g \rightarrow g f g: Y \rightarrow X
\end{aligned}
$$

We say that this future 2-equivalence is coherent if the following axioms hold:

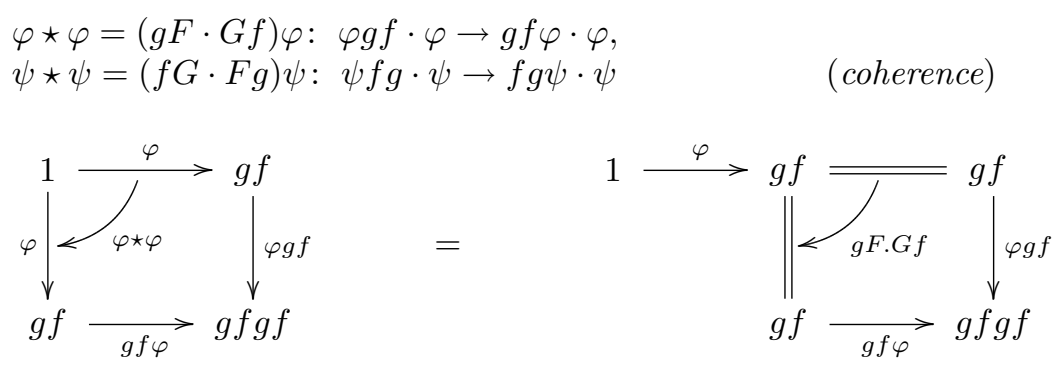

The diagram above refers to the first condition. (See 7.3 for the modification $\varphi \star \varphi$, a graded composite; see 7.2 for the whisker composition of the modification $g F \cdot G f$ with the lax natural transformation $\varphi$ ). A future 2-equivalence between ordinary categories (with trivial cells) amounts to an ordinary future equivalence, as defined in Part I, since its comparisons reduce to identities.

Both notions are reflexive and symmetric; the first - future 2-equivalence - is also transitive (as we prove in 3.2). Moreover (having chosen the arrow of the comparison cells of lax natural transformations, in 2.2), the arrow of the comparison cells $F, G$ in the previous definition cannot be inverted, if we want the result of Thm. 3.4 (see the note at the end of the proof).

A property (making sense in a 2-category, or for a 2-category) will be said to be future 2-invariant if it is preserved by future 2-equivalences; an elementary example will be future 2-contractibility (3.5). A future 2-equivalence between ordinary categories amounts to a future equivalence (1.3).

A coherent local adjunction $f \dashv g(2.4)$ with invertible counit $\varepsilon: f g \rightarrow 1$ and invertible comparisons $L: \varepsilon f \cdot f \eta \rightarrow 1 f$ and $R: 1 g \rightarrow g \varepsilon \cdot \eta g$ amounts to a coherent 
future 2-equivalence with invertible unit $\psi$ and invertible comparisons, letting:

$$
\begin{array}{llrl}
\varphi & =\eta, & \psi & =\varepsilon^{-1}, \\
F=(\psi f \cdot L)^{-1}: \psi f \rightarrow f \varphi, & G & =(g \psi \cdot R)^{-1}: \varphi g \rightarrow g \psi .
\end{array}
$$

This case, a 'split' future 2-equivalence, will be treated later (3.3).

Dually, a past 2-equivalence $(f, g ; \varphi, \psi ; F, G)$ has

$$
\begin{aligned}
& f: X \rightleftarrows Y: g, \\
& \varphi: g f \rightarrow 1_{X}: X \rightarrow X, \\
& F: f \varphi \rightarrow \psi f: f g f \rightarrow f: X \rightarrow Y, \\
& G: g \psi \rightarrow \varphi g: g f g \rightarrow g: Y \rightarrow X
\end{aligned}
$$

and is coherent if:

$$
\begin{aligned}
& (G f \cdot g F) \varphi=\varphi \star \varphi: g f \varphi \cdot \varphi \rightarrow \varphi g f \cdot \varphi \\
& (F g \cdot f G) \psi=\psi \star \psi: f g \psi \cdot \psi \rightarrow \psi f g \cdot \psi
\end{aligned}
$$

Future 2-equivalences, being linked with (locally) reflective sub-2-categories and idempotent 2-monads (3.3), will generally be given priority with respect to the dual case (related with coreflective sub-2-categories and 2-comonads). The cell-dual notion, an op-future 2-equivalence, will only be considered marginally; it has op-lax natural transformations $\varphi: 1_{X} \rightarrow g f, \psi: 1_{Y} \rightarrow f g$ directed the same way but having opposite comparison cells (2.2) and triangular comparisons directed the other way round $(F: f \varphi \rightarrow \psi f, G: g \psi \rightarrow \varphi g)$. Finally, notice that (in contrast with 2.5) a future 2-equivalence does not induce a future equivalence (nor functors) at the level of hom-categories.

\subsection{Composition}

Future 2-equivalences can be composed (much in the same way as local adjunctions, in [15], I.7.3), which shows that being future equivalent 2-categories is an equivalence relation.

In fact, after $(f, g ; \varphi, \psi ; F, G)$ (as in (26)), let a second future 2-equivalence be given

$$
\begin{gathered}
h: Y \rightleftarrows Z: k, \\
\vartheta: 1_{Y} \rightarrow k h: Y \rightarrow Y, \quad \zeta: 1_{Z} \rightarrow h k: Z \rightarrow Z, \\
H: \zeta h \rightarrow h \vartheta: h \rightarrow h k h: Y \rightarrow Z, \quad K: \vartheta k \rightarrow k \zeta: k \rightarrow k h k: Z \rightarrow Y .
\end{gathered}
$$

Their composite is defined as follows:

$$
h f: X \rightleftarrows Z: g k,
$$

$$
\begin{aligned}
& g \vartheta f \cdot \varphi: 1_{X} \rightarrow g k \cdot h f, \quad h \psi k \cdot \zeta: 1_{Z} \rightarrow h f \cdot g k, \\
& L:(h \psi k \cdot \zeta) h f \rightarrow h f(g \vartheta f \cdot \varphi): h f \rightarrow h f \cdot g k \cdot h f: X \rightarrow Z, \\
& R:(g \vartheta f \cdot \varphi) g k \rightarrow g k(h \psi k \cdot \zeta): g k \rightarrow g k \cdot h f \cdot g k: Z \rightarrow X,
\end{aligned}
$$

where the modifications $L$ and $R$ are given by the following two pastings, in the 2-categories $\operatorname{Lnt}(X, Z)$ and $\operatorname{Lnt}(Z, X)$ of 2-functors, lax natural transformations 
and modifications (7.2)
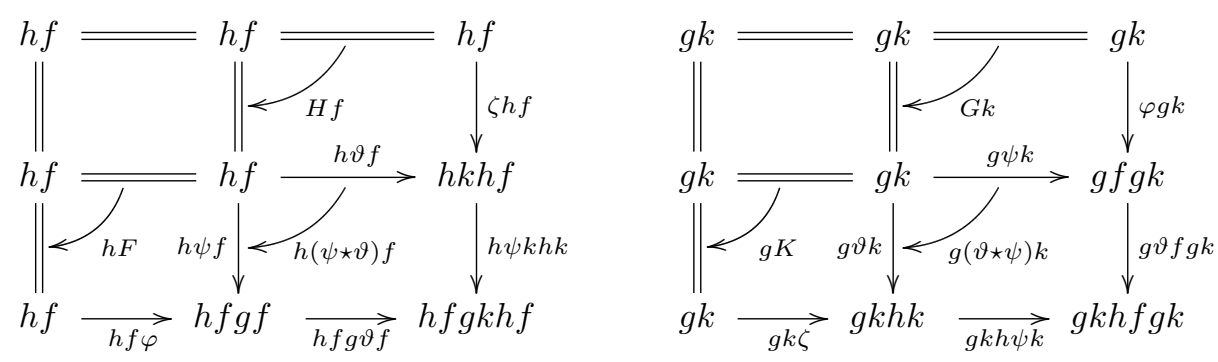

On the other hand, the coherent case seems not to be closed under composition. (Note that every future 2-equivalence can be factorised into two coherent ones, by Thm. 3.4.)

\subsection{Future 2-retracts}

As in the 1-dimensional case (I.2), there is an important instance of future 2equivalence, which generates the general situation (as will be shown in Thm. 3.4).

A split future 2-equivalence of $X$ onto $X_{0}$ ( or of $X_{0}$ into $X$ ) will be a coherent future 2 -equivalence $(p, i ; \eta, i ; 1,1)$ where the unit $1_{X_{0}} \rightarrow p i$ and both comparisons are identities

$$
\begin{array}{cll}
p: X \rightleftarrows X_{0}: i, & \eta: 1_{X} \rightarrow i p, & p i=1_{X_{0}}, \\
p \eta=1_{p}, & \eta i=1_{i}, & \\
1_{\eta}=\eta \star \eta: \eta i p \cdot \eta \rightarrow i p \eta \cdot \eta & & \text { (coherence). }
\end{array}
$$

This equivalence $(p, i ; \eta, i ; 1,1)$ is a split epi in the category of future 2 -equivalences, with section $(i, p ; 1, \eta ; 1,1)$ (use the composition diagram (33)). We shall view $i$ as an inclusion and $X_{0}$ as a sub-2-category of $X$; it is easy to see that $X_{0}$ is locally full in $X$ (but not necessarily full, as shown by the examples of Section 6 ). Indeed, every $X$-cell $\alpha: a \rightarrow b: x \rightarrow x^{\prime}$ between maps of $X_{0}$ necessarily belongs to the latter, since the lax natural transformation $\eta: 1_{X} \rightarrow i p$ gives the following equality (axiom (lnt.3) in 2.2)

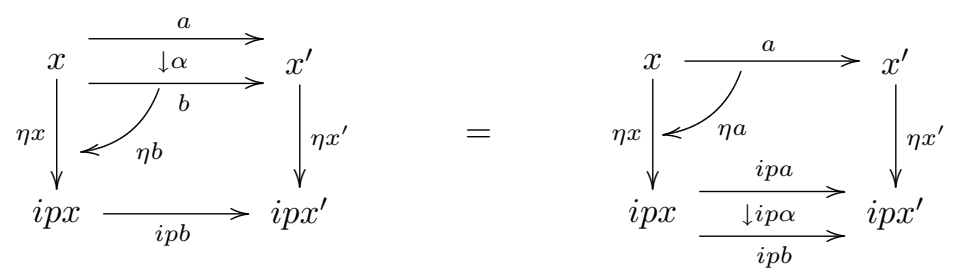

where $\eta x, \eta x^{\prime}$ are identity maps and $\eta a, \eta b$ are identity cells (by $\eta i=1_{i}$ ).

Equivalently, we have a strictly coherent local adjunction $p \dashv i$ with unit $\eta: 1_{X} \rightarrow$ $i p$, where the counit is an identity (as well as both comparisons, cf. 2.4). Thus, $p$ will be called the local reflector of the embedding $i$. 
Equivalently again, one can assign a strictly idempotent coherent local monad $(e, \eta)$ on $X$, i.e., a 2-endofunctor $e$ and a lax natural transformation $\eta$ such that

$$
\begin{array}{ll}
e: X \rightarrow X, & \eta: 1_{X} \rightarrow e, \\
e e=e, & e \eta=1_{e}=\eta e, \quad \eta \star \eta=1_{\eta} .
\end{array}
$$

Indeed, given $(i, p ; \eta)$, we take $e=i p$; given $(e, \eta)$, we factor $e=i p$ splitting $e$ through the sub-2-category $X_{0}$ of $X$ containing the objects, arrows and cells which $e$ leaves fixed.

Dually, a split past equivalence of $X_{0}$ into $X$ (or of $X$ onto $X_{0}$ ) is a coherent past equivalence $(p, i ; \varepsilon, 1 ; 1,1)$ where the counit $p i \rightarrow 1_{P}$ and both comparisons are identities

$$
\begin{array}{lll}
p: X \rightleftarrows X_{0}: i, & \varepsilon: i p \rightarrow 1_{X}, & p i=1_{X_{0}}, \\
p \varepsilon=1_{p}, & \varepsilon i=1_{i}, & 1_{e}=\varepsilon \star \varepsilon: \varepsilon \cdot i p \varepsilon \rightarrow \varepsilon \cdot \varepsilon i p .
\end{array}
$$

Then, $X_{0}$ will be said to be a past retract of $X$, with local coreflector $p$ (locally right adjoint to the inclusion, with trivial unit and comparisons).

3.4. Theorem (Future 2-equivalence and future 2-retracts)

(a) A future 2-equivalence $(f, g ; \varphi, \psi ; F, G)$ between $X$ and $Y$ (3.1) has a canonical factorisation into two split future 2-equivalences

$$
X \underset{p}{\stackrel{i}{\rightleftarrows}} W \underset{j}{\stackrel{q}{\rightleftarrows}} Y, \quad \eta: 1_{W} \longrightarrow i p, \quad \eta^{\prime}: 1_{W} \longrightarrow j q,
$$

where $X$ and $Y$ are future 2-retracts of $W$ (the graph of the given future 2-equivalence).

(b) Two 2-categories are future 2-equivalent if and only if they are future 2-retracts of a third.

(c) A property is future 2-invariant if and only if it is preserved by all embeddings of future 2-retracts, as well as by their local reflectors.

Proof. (a). First, we construct the 2-category $W$, enriching the construction of I.2.5 for 1-dimensional categories.

(i) An object is a six-tuple $(x, y ; u, v ; U, V)$ containing two objects, two maps and two cells, such that:

$$
\begin{array}{ll}
u: x \rightarrow g y \quad(\text { in } X), & v: y \rightarrow f x \\
U: \varphi x \rightarrow g v \cdot u, & V: \psi y \rightarrow f u \cdot v,
\end{array}
$$
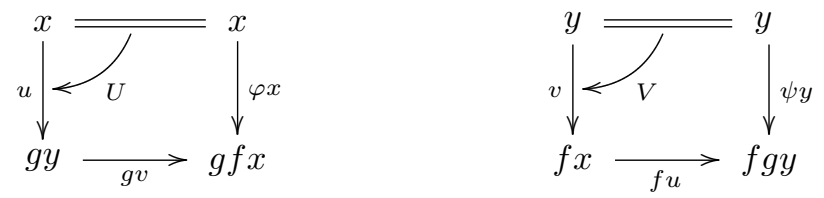
(ii) A morphism is a four-tuple $(a, b ; A, B):(x, y ; u, v ; U, V) \rightarrow\left(x^{\prime}, y^{\prime} ; u^{\prime}, v^{\prime} ; U^{\prime}, V^{\prime}\right)$ with two maps and two cells, such that:

$$
\begin{array}{lll}
a: x \rightarrow x^{\prime} & (\text { in } X), & b: y \rightarrow y^{\prime} \\
A: u^{\prime} a \rightarrow g b \cdot u, & B: v^{\prime} b \rightarrow f a \cdot v,
\end{array}
$$
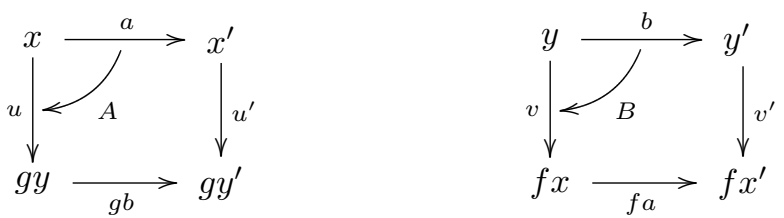

(a coherence condition can be added; but this is not necessary). A cell between parallel maps $(\alpha, \beta):(a, b ; A, B) \rightarrow\left(a^{\prime}, b^{\prime} ; A^{\prime}, B^{\prime}\right)$ is a pair such that:

$$
\left.\begin{array}{lll}
\alpha: a \rightarrow a^{\prime} & (\text { in } X), & \beta: b \rightarrow b^{\prime} \\
(g \beta \cdot u) A=A^{\prime} \cdot u^{\prime} \alpha, & (f \alpha \cdot v) B=B^{\prime} \cdot v^{\prime} \beta,
\end{array} \quad \text { (in } Y\right)
$$
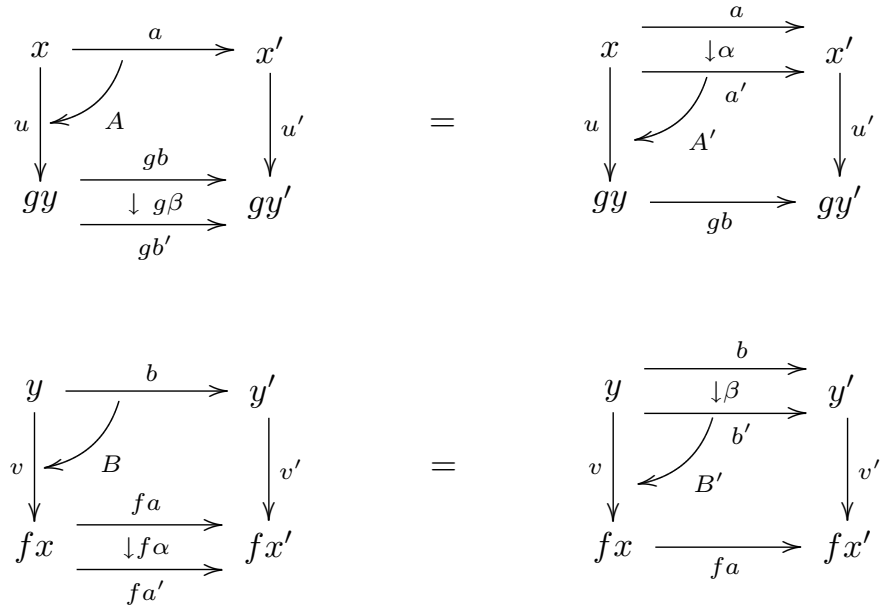

(iii) The composition of arrows is as follows (it is easy to see that it is 'categorical')

$$
\left(a^{\prime}, b^{\prime} ; A^{\prime}, B^{\prime}\right) \cdot(a, b ; A, B)=\left(a^{\prime} a, b^{\prime} b ; A \otimes_{h} A^{\prime}, B \otimes_{h} B^{\prime}\right),
$$
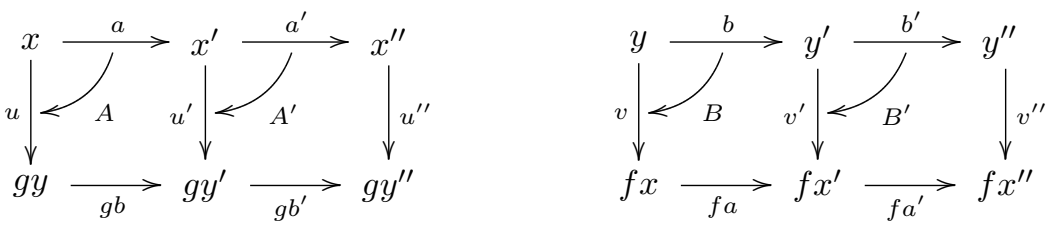
(iv) The main and secondary compositions of cells are defined component-wise (and satisfy the axioms of 2-categories, with the obvious identities)

$$
\left(\alpha^{\prime}, \beta^{\prime}\right) \cdot(\alpha, \beta)=\left(\alpha^{\prime} \alpha, \beta^{\prime} \beta\right), \quad(\gamma, \delta) \circ(\alpha, \beta)=(\gamma \circ \alpha, \delta \circ \beta) .
$$

The construction of the 2-category $W$ is now completed. We have a split future 2-equivalence of $X$ into $W$

$$
\begin{aligned}
& i: X \rightleftarrows W: p, \quad \eta: 1_{W} \rightarrow i p, \\
& i(x)=\left(x, f x ; \varphi x, 1_{f} x ; 1_{\varphi x}, F x: \psi f x \rightarrow f \varphi x\right), \\
& i\left(a: x \rightarrow x^{\prime}\right)=\left(a, f a ; \varphi a: \varphi x^{\prime} \cdot a \rightarrow g f a \cdot \varphi x, 1_{f a}\right) \text {, } \\
& i\left(\alpha: a \rightarrow a^{\prime}\right)=(\alpha, f \alpha), \\
& p(x, y ; u, v ; U, V)=x, \quad p(a, b ; A, B)=a, \quad p(\alpha, \beta)=\alpha, \\
& \eta(x, y ; u, v ; U, V)=\left(1_{x}, v ; U: \varphi x \rightarrow g v \cdot u, 1_{v}\right):(x, y ; u, v ; U, V) \rightarrow i(x) \text {, } \\
& \eta(a, b ; A, B)=\left(1_{a}, B\right):\left(a, v^{\prime} b ; U^{\prime} a \cdot g v^{\prime} A, B\right) \rightarrow(a, f a \cdot v ; \varphi a \cdot g f a U, 1), \\
& (x, y ; u, v ; U, V) \longrightarrow\left(x^{\prime}, y^{\prime} ; u^{\prime}, v^{\prime} ; U^{\prime}, V^{\prime}\right) \\
& \left(1_{x}, v ; U, 1_{v}\right) \mid \underset{\eta(a, b ; A, B)}{\downarrow}\left(1_{x}^{\prime}, v^{\prime} ; U^{\prime}, 1_{v}^{\prime}\right) \\
& \left(x, f x ; \varphi x, 1_{f x} ; 1_{\varphi x}, F x\right) \underset{\left(a, f a ; \varphi a, 1_{f a}\right)}{\longrightarrow}\left(x^{\prime}, f x^{\prime} ; \varphi x^{\prime}, 1_{f x^{\prime}} ; 1_{\varphi x}, F x^{\prime}\right)
\end{aligned}
$$

The correctness of the definitions of $i$ and $\eta$ is easily verified; for instance, the coherence of the lax natural transformation $\eta$ with a $W$-cell $(\alpha, \beta)$ (property (lnt.3) of 2.2) follows from the definition of a cell, in (42). The relations $p i=1_{W}, \eta i=$ $1_{i}, p \eta=1_{p}$ are plain. We also have $\eta \star \eta=1_{\eta}$ (independently of the coherence of the original future 2-equivalence)

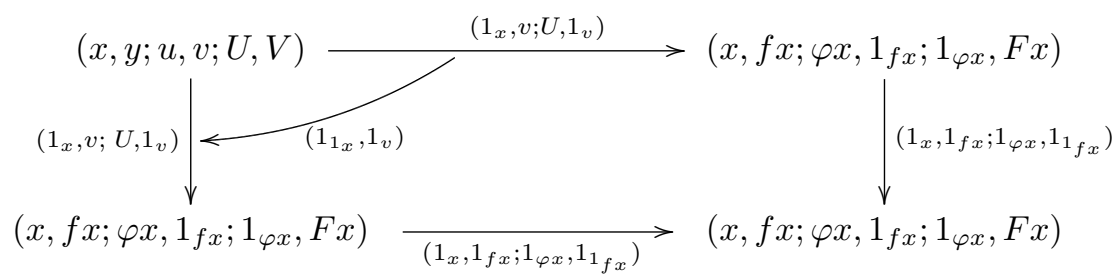

Symmetrically, there is a split future 2-equivalence of $Y$ into $W$

$$
\begin{gathered}
j: Y \rightleftarrows W: q, \quad \eta^{\prime}: 1_{W} \rightarrow j q, \\
j(y)=\left(g y, y ; 1_{g y}, \psi y ; G y: \varphi g y \rightarrow g \psi y, 1_{\psi y}\right), \\
j\left(b: y \rightarrow y^{\prime}\right)=\left(g b, b ; 1_{g b}, \psi b: \psi y^{\prime} \cdot b \rightarrow f g b \cdot \psi y\right), \quad j(B)=(g B, B), \\
q(x, y ; u, v ; U, V)=y, \quad q(a, b ; A, B)=b, \quad q(A, B)=B,
\end{gathered}
$$


Homology, Homotopy and Applications, vol. 8(1), 2006

$$
\begin{aligned}
& \eta^{\prime}(x, y ; u, v ; U, V)=\left(u, 1_{y} ; 1_{u}, V\right):(x, y ; u, v ; U, V) \rightarrow j(y), \\
& \eta^{\prime}(a, b ; A, B)=\left(A, 1_{b}\right):\left(u^{\prime} a, b ; A, V^{\prime} b \cdot f u^{\prime} B\right) \rightarrow(g b \cdot u, b ; 1, \psi b \cdot f g b V) .
\end{aligned}
$$

Now, composing these two equivalences as in (39) (cf. (32) and (33)), gives back the original future 2-equivalence $(f, g ; \varphi, \psi ; F, G)$

$$
\begin{aligned}
& \left(q, j ; \eta^{\prime}, 1 ; 1,1\right) \cdot(i, p ; 1, \eta ; 1,1)=\left(q i, p j ; p \eta^{\prime} i, q \eta j ; q\left(\eta \star \eta^{\prime}\right) i, p\left(\eta^{\prime} \star \eta\right) j\right), \\
& \quad q i(x)=f(x), \quad q i(a)=f(a), \quad q i(A)=f(A), \\
& p \eta^{\prime} i: 1_{X} \rightarrow p j \cdot q i, \\
& p \eta^{\prime} i(x)=p \eta^{\prime}\left(x, f x ; \varphi x, 1_{f x} ; 1_{\varphi x}, F x\right)=p\left(\varphi x, 1_{f x} ; 1_{\varphi x}, F x\right)=\varphi x, \\
& p \eta^{\prime} i(a)=p \eta^{\prime}\left(a, f a ; \varphi a, 1_{f a}\right)=p\left(\varphi a, 1_{f a}\right)=\varphi a, \\
& q\left(\eta \star \eta^{\prime}\right) i: f \varphi \rightarrow \psi f, \\
& q\left(\eta \star \eta^{\prime}\right) i(x)=q\left(\eta\left(\eta^{\prime} i(x)\right)\right)=q\left(\eta\left(\varphi x, 1_{f x} ; 1_{\varphi x}, F x\right)\right)=q\left(1_{\varphi x}, F x\right) \\
& =F x .
\end{aligned}
$$

Finally, (b) and (c) follow immediately from (a), by composing future 2-equivalences (3.2).

We also note that the proof shows the 'necessity' of the previous choices for the direction of cells (once we have fixed it, for lax natural transformations). Indeed, the direction of the cell $A$ in (41) must agree with the direction of $\varphi a: \varphi x^{\prime} \cdot a \rightarrow g f a \cdot \varphi x$ in (46); but then, because of (48), the arrow of $U$ is also fixed; finally, (51) determines the arrow of $G y$.

\subsection{Future 2-contractible 2-categories}

We say that a 2-category $X$ is future 2-contractible if the 2-functor $p: X \rightarrow \mathbf{1}$ with values in the singleton 2 -category (one object $\star$ and its identities) is a future 2-equivalence.

This means that we have a 2-functor $i: \mathbf{1} \rightarrow X$ (i.e., an object $x_{0}=i(\star)$ of $X$ ), with a lax natural transformation $\eta$ and a modification $G$

$$
\begin{aligned}
& p: X \rightleftarrows 1: i, \\
& \eta: 1_{X} \rightarrow i p: X \rightarrow X, \quad G: \eta i \rightarrow 1_{i}: i \rightarrow i: \mathbf{1} \rightarrow X ;
\end{aligned}
$$

note that $G$ merely amounts to a cell $G_{0}=G(\star): \eta x_{0} \rightarrow i d\left(x_{0}\right)$. (The axiom (mdf), in 2.3 , is trivially satisfied, since $\mathbf{1}$ has precisely one arrow, which is an identity.)

In this situation, we also say that $X$ is future 2-contractible to the object $x_{0}$. Notice that the latter is not determined up to isomorphism (as shown at the end of this subsection).

We say that $X$ is split future 2-contractible if $p: X \rightarrow \mathbf{1}$ is a split future 2-equivalence onto 1 , i.e., a coherent future 2-equivalence with comparison $G=1$. This amounts to a strictly coherent local adjunction $p \dashv i$ with unit $\eta$ (and counit 
$p i=1 ;$ cf. 2.4)

$$
\begin{array}{ll}
p: X \rightleftarrows \mathbf{1}: i, & \eta: 1_{X} \rightarrow i p: X \rightarrow X, \\
\eta i=1_{i}, & \eta(\eta x)=1_{\eta x} .
\end{array}
$$

We have already seen, in 2.6, that Rel $\mathbf{A b}$ is split future 2-contractible (to the object 0 ) and split past 2-contractible (to the same object); moreover, the same is true in the cell-dual sense, with respect to op-local adjunctions. There are no other split solutions. Indeed, if $(i, \eta)$ is 1 , the following cell (for an arbitrary $a: x \rightarrow i(\star)$ ) shows that every component $\eta x: x \rightarrow i(\star)$ must be the greatest relation $x \rightarrow i(\star)$

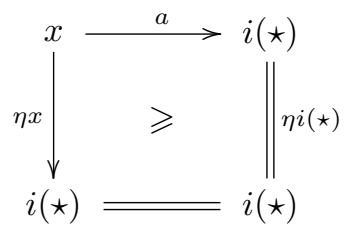

and then $\eta i=1$ shows that $i(\star)=0$.

Similarly, the 2-category Rel Set is split future 2-contractible to precisely two objects (up to isomorphism): the empty set and the singleton.

\subsection{Proposition}

In order that the category $X$ be split future 2-contractible to an object $x_{0}$, it suffices that in the latter there be, for every object $x$, an arrow $\eta x: x \rightarrow x_{0}$ which is terminal in the category $X\left(x, x_{0}\right)$ and such that $\eta x_{0}=i d\left(x_{0}\right)$.

Proof. Let $i: \mathbf{1} \rightarrow X, i(\star)=x_{0}$. For every arrow $a: x \rightarrow x^{\prime}$ in $X$, let $\eta a: \eta x^{\prime} \cdot a \rightarrow$ $\eta x$ be the unique cell to the terminal arrow $\eta x: x \rightarrow x_{0}$. Plainly, this defines a lax natural transformation $\eta: 1 \rightarrow i p(2.2)$. Again, let $G(\star): 1_{x_{0}} \rightarrow \eta x_{0}$ be the unique cell to the terminal arrow $\eta x_{0}: x_{0} \rightarrow x_{0}$; this defines a modification $G: \eta i \rightarrow$ $1_{i}: i \rightarrow i: \mathbf{1} \rightarrow X$. The condition $\eta i=1_{i}$ is already assumed, and $\eta(\eta x): \eta x \rightarrow \eta x$ necessarily coincides with $1_{\eta x}$.

\section{Two-dimensional models}

We see here how a past 2-retract and a future 2-retract of a 2-category generate a global 2-dimensional model (4.3). Then, we study properties of objects, invariant up to future 2-equivalence, which will be of use to construct minimal models of 2-categories $(4.4,4.5)$.

\subsection{Injective 2-models}

A future 2-embedding of $E$ into $X$ will be a future 2-equivalence $\left(f, g ; \eta_{E}, \eta\right)$ where the comparison cells are identities, $f$ is a locally full 2-embedding and some 
additional properties hold, as follows:

$$
f: E \rightleftarrows X: g
$$

$$
\begin{array}{ll}
\eta_{E}: 1_{E} \rightarrow g f: E \rightarrow E, & \eta: 1_{X} \rightarrow f g: X \rightarrow X, \\
\eta f=f \eta_{E}: f \rightarrow f g f: E \rightarrow X, & \eta_{E} g=1=g \eta: g \rightarrow g f g: X \rightarrow E, \\
\eta \star \eta=1_{\eta}: \eta \rightarrow \eta, &
\end{array}
$$

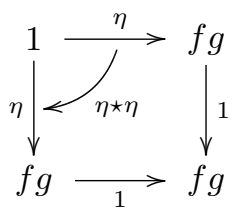

In particular, $g f g=g$, so that $g f$ and $f g$ are idempotent endofunctors. Moreover, $\eta_{E}$ is determined by $\eta$ (the main unit) and $\eta_{E} \star \eta_{E}=1_{\eta_{E}}$ holds as well, so that our future 2-equivalence is strictly coherent (3.1).

Dually, we have the notion of a past 2-embedding. Combining both aspects, the 2-category $E$ is made an injective 2-model of $X$ by assigning a pf-2-embedding of $E$ into $X$, i.e., a pair formed of a past 2-embedding $\left(f, g^{-} ; \varepsilon_{E}, \varepsilon\right)$ and a future 2-embedding $\left(f, g^{+} ; \eta_{E}, \eta\right)$ sharing the same locally full 2-embedding $f: E \rightarrow X$ :

$$
\begin{aligned}
& f: E \leftrightarrows X: g^{-}, g^{+}, \\
& \varepsilon_{E}: g^{-} f \rightarrow 1_{E}, \quad \varepsilon: f g^{-} \rightarrow 1_{X}, \\
& f \varepsilon_{E}=\varepsilon f: f g^{-} f \rightarrow f, \quad g^{-} \varepsilon=1=\varepsilon_{E} g^{-}: g^{-} f g^{-} \rightarrow g^{-}, \quad \varepsilon \star \varepsilon=1_{\varepsilon}, \\
& \eta_{E}: 1_{E} \rightarrow g^{+} f, \quad \eta: 1_{X} \rightarrow f g^{+}, \\
& \eta f=f \eta_{E}: f \rightarrow f g^{+} f, \quad g^{+} \eta=1=\eta_{E} g^{+}: g^{+} \rightarrow g^{+} f g^{+}, \quad \eta \star \eta=1_{\eta},
\end{aligned}
$$
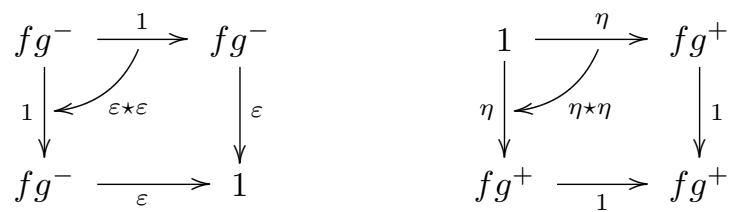

\subsection{Pf-presentations}

We introduce now a second structure which combines past and future, and will produce an injective 2-model.

A pf-2-presentation of the category $X$ will be a diagram consisting of a past 2 -retract $P$ and a future 2-retract $F$ of $X$ (3.3; both are locally full sub-2-categories)

$$
P \underset{p^{-}}{\stackrel{i^{-}}{\gtrless}} X \underset{i^{+}}{\stackrel{p^{+}}{\gtrless}} F
$$

$$
\begin{aligned}
& \varepsilon: i^{-} p^{-} \rightarrow 1_{X} \quad\left(p^{-} i^{-}=1, p^{-} \varepsilon=1, \varepsilon i^{-}=1, \varepsilon \star \varepsilon=1_{\varepsilon}\right), \\
& \eta: 1_{X} \rightarrow i^{+} p^{+} \quad\left(p^{+} i^{+}=1, p^{+} \eta=1, \eta i^{+}=1, \eta \star \eta=1_{\eta}\right) \text {. }
\end{aligned}
$$


We have thus two strictly coherent local adjunctions $i^{-} \dashv p^{-}, p^{+} \dashv i^{+}$.

Recall that $P$ and $F$ are locally full sub-2-categories of $X$ (3.3). We form now a locally full sub-2-category $E$, which will be called the injective 2-model of $X$ generated by the pf-2-presentation (61) - and will be proved to be such a model (4.3). Its objects belong to $P_{0} \cup F_{0}$ (i.e., $\mathrm{Ob} P \cup \mathrm{Ob} F$ ), while its arrows are generated by:

(a) the arrows of $P$ and $F$,

(b) the components $\varepsilon x: i^{-} p^{-} x \rightarrow x$, for $x \in F_{0}$, and $\eta x: x \rightarrow i^{+} p^{+} x$, for $x \in P_{0}$.

Note that all the components of $\varepsilon, \eta$ on items of $E$ live in $E$. In fact, for an object $x \in P_{0} \cup F_{0}$, it suffices to consider the condition (b), together with the properties $\varepsilon x=1_{x}$ for $x \in P_{0}$ and $\eta x=1_{x}$ for $x \in F_{0}$ (and condition (a)). Secondly, for a map $a: x \rightarrow x^{\prime}$ in $E$, the thesis follows from the fact that both cells $\varepsilon a$ and $\eta a$ have a domain-arrow and a codomain-arrow in $E$ (see the diagrams below), which is locally full in $X$
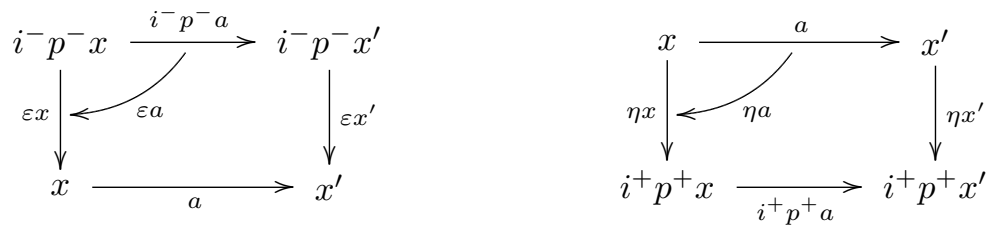

4.3. Theorem (Pf-2-presentations and injective 2-models)

Given a pf-2-presentation of the 2-category $X$ (written as in (61)), let $E$ be the locally full sub-2-category of $X$ described above (4.2) and $f$ its embedding in $X$.

(a) These data can be uniquely completed to the left diagram below, with (four) commutative squares

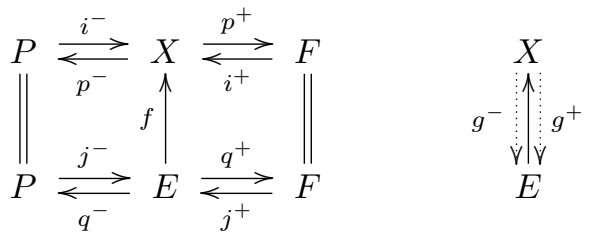

Moreover:

(b) there is a unique lax natural transformation $\varepsilon_{E}: j^{-} q^{-} \rightarrow 1_{E}$ such that $f \varepsilon_{E}=\varepsilon f$

(c) there is a unique lax natural transformation $\eta_{E}: 1_{E} \rightarrow j^{+} q^{+}$such that $f \eta_{E}=\eta f$

(d) these transformations make the lower row into a pf-2-presentation of $E$;

(e) letting $g^{\alpha}=j^{\alpha} p^{\alpha}: X \rightarrow E(\alpha= \pm)$, we get a pf-2-embedding

$$
\left(f, g^{-}, g^{+} ; \varepsilon_{E}, \varepsilon, \eta_{E}, \eta\right): E \rightarrow X,
$$

making $E$ an injective 2-model of $X$ (generated by the presentation).

Proof. (a) First, we (must) take $j^{+}: F \subset E$ (so that $f j^{+}=i^{+}$) and $q^{+}=p^{+} f: E \rightarrow$ $F$. Symmetrically, we take $j^{-}: P \subset E$ and $q^{-}=p^{-} f$. 
Now, we prove (b) to (d), completing the lower row of diagram (63) to a pf-2-presentation of $E$, as claimed. On the right side, we already know that $q^{+} j^{+}=$ $p^{+} i^{+}=1_{F}$ and we have seen, at the end of 4.2 , that all the components of $\eta f: f \rightarrow$ $i^{+} p^{+} f: E \rightarrow X$ belong to $E$. There is thus a unique lax natural transformation $\eta_{E}: 1_{E} \rightarrow j^{+} q^{+}$such that $f \eta_{E}=\eta f$; plainly, $\eta_{E} j^{+}=1$ and $q^{+} \eta_{E}=1$. Similarly for $\varepsilon_{E}: j^{-} q^{-} \rightarrow 1_{E}$.

(e) Then, we define $g^{\alpha}=j^{\alpha} p^{\alpha}: X \rightarrow E$ and observe that

$$
f g^{+}=f j^{+} p^{+}=i^{+} p^{+}, \quad g^{+} f=j^{+} p^{+} f=j^{+} q^{+} .
$$

Therefore, we can take the lax natural transformations

$$
\eta: 1_{X} \rightarrow i^{+} p^{+}=f g^{+}, \quad \eta_{E}: 1_{E} \rightarrow j^{+} q^{+}=g^{+} f,
$$

as the units of the pf-2-embedding $f: E \leftrightarrows X: g^{\alpha}$; in fact, the relations:

$$
\eta f=f \eta_{E}, \quad g^{+} \eta=1=\eta_{E} g^{+}, \quad \eta \star \eta=1_{\eta},
$$

are already known, or come from $g^{+} \eta=j^{+} p^{+} \eta=1, \eta_{E} g^{+}=\eta_{E} j^{+} p^{+}=1$. Similarly for the counits.

\subsection{Future 2-regularity}

A point $x$ in the 2-category $X$ will be said to be $V_{2}^{+}$-regular if it satisfies the condition (i), $\mathrm{O}_{2}^{+}$-regular if it satisfies (ii) and future 2-regular if it satisfies both:

(i) for any pair of 2-cells $\alpha_{i}: a \rightarrow a_{i}: x \rightarrow x^{\prime} \quad(i=1,2$; see the left diagram below), there exists a pair of double cells $\xi_{i}$ such that $\alpha_{1} \otimes_{v} \xi_{1}=\alpha_{2} \otimes_{v} \xi_{2}$,

(ii) given three 2-cells $\alpha: a \rightarrow a^{\prime}: x \rightarrow x^{\prime}$ and $\alpha_{i}: a^{\prime} \rightarrow a^{\prime \prime}: x \rightarrow x^{\prime}(i=1,2$; see the right diagram below) such that $\alpha \otimes_{v} \alpha_{1}=\alpha \otimes_{v} \alpha_{2}$, there exists a 2-cell $\xi$ (with boundary as below) such that $\alpha_{1} \otimes_{v} \xi=\alpha_{2} \otimes_{v} \xi$
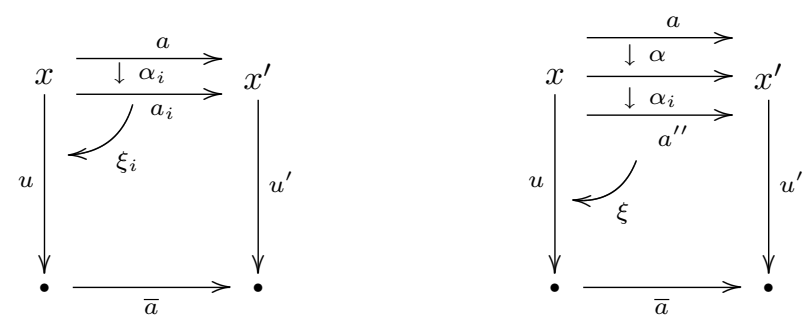

On the other hand, we shall say that $x$ is $V_{2}^{+}$-branching if it is not $V_{2}^{+}$-regular; that it is $\mathrm{O}_{2}^{+}$-branching if it is not $\mathrm{O}_{2}^{+}$-regular; that is a future 2-branching if it falls in (at least) one of the previous cases, i.e., if it is not future 2-regular. Dually, we have $V_{2}^{-}$-regular, $\mathrm{O}_{2}^{-}$-regular, past 2-regular points and the corresponding branching points.

4.5. Theorem (Future 2-equivalences and regular points)

Given a future 2-equivalence $f: X \rightleftarrows Y: g$, with lax natural transformations $\varphi: 1 \rightarrow g f, \psi: 1 \rightarrow f g$ and comparisons $F: \psi f \rightarrow f \varphi, G: \varphi g \rightarrow g \psi$, we have:

(a) the functors $f$ and $g$ preserve $V_{2}^{+}$-regular, $O_{2}^{+}$-regular and future 2-regular points, 
Homology, Homotopy and Applications, vol. 8(1), 2006

(b) the functors $f$ and $g$ preserve $V_{2}^{+}$-branching, $\mathrm{O}_{2}^{+}$-branching and future 2branching points (i.e., reflect $V_{2}^{+}$-regular, $\mathrm{O}_{2}^{+}$-regular and future 2-regular points).

Proof. The index $i$ always takes values 1,2 .

(a) Suppose that $x$ is $V_{2}^{+}$-regular (in $X$ ); we must prove that $f x$ is also (in $Y$ ). Given a pair of 2-cells $\beta_{i}: b \rightarrow b_{i}: f x \rightarrow y$ in $Y$, as in the right diagram below, there exists in $X$ a pair of double cells $\xi_{i}$ as in the left diagram, such that $\left(g \beta_{i} \cdot \varphi x\right) \otimes_{v} \xi_{i}=\xi$ (independent of $i$ )
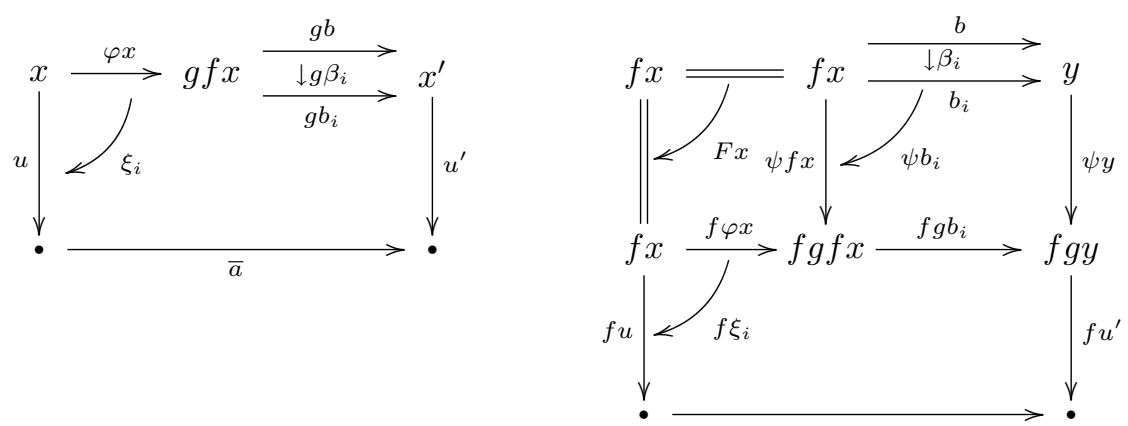

Then, in the right diagram above, the double cells $\eta_{i}=\left(F x \otimes_{h} \psi b_{i}\right) \otimes_{v} f \xi_{i}$ have the same vertical composition with $\beta_{i}$

$$
\begin{aligned}
& \beta_{i} \otimes_{v} \eta_{i}=\beta_{i} \otimes_{v}\left(F x \otimes_{h} \psi b_{i}\right) \otimes_{v} f \xi_{i} \\
& =\left(F x \otimes_{h}\left(\beta_{i} \otimes_{v} \psi b_{i}\right)\right) \otimes_{v} f \xi_{i}=\left(F x \otimes_{h}\left(\psi b \otimes_{v} f g \beta_{i}\right)\right) \otimes_{v} f \xi_{i} \\
& =\left(F x \otimes_{h} \psi b\right) \otimes_{v}\left(f g \beta_{i} \cdot f \varphi x\right) \otimes_{v} f \xi_{i}=\left(F x \otimes_{h} \psi b\right) \otimes_{v} f \xi .
\end{aligned}
$$

Second, suppose that $x$ is $O_{2}^{+}$-regular in $X$, and let us prove the same of $f x$ in $Y$. Take, in the right diagram below, three 2-cells $\beta: b \rightarrow b^{\prime}: f x \rightarrow y$ and $\beta_{i}: b^{\prime} \rightarrow b^{\prime \prime}$, so that $\beta \otimes_{v} \beta_{1}=\beta \otimes_{v} \beta_{2}$
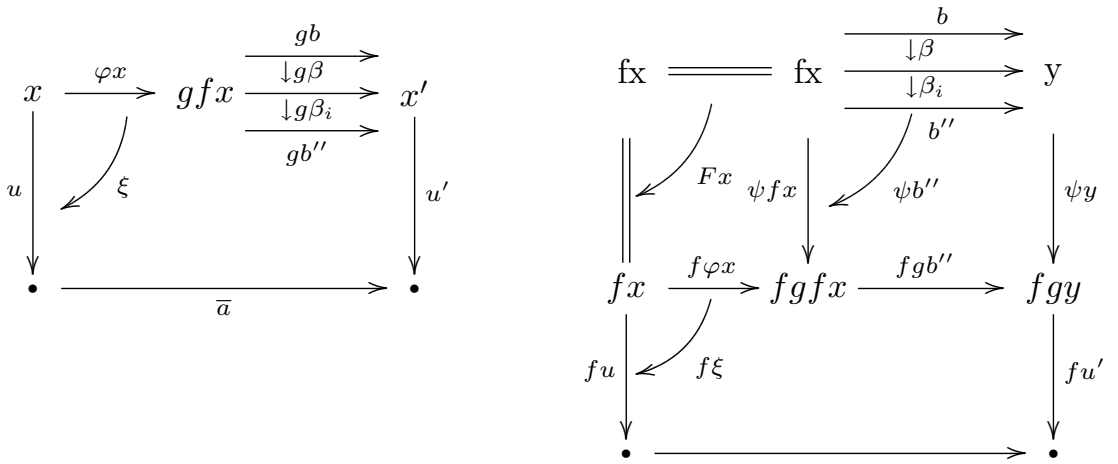
Then, in $X$, the composite $g \beta \cdot \varphi x \otimes_{v} g \beta_{i} \cdot \varphi x$ (which 'starts' at $x$ ) does not depend on $i$, and there exists a 2 -cell $\xi$ such that $\left(g \beta_{i} \cdot \varphi x\right) \otimes_{v} \xi=\bar{\xi}$ (independent of $i$ ). One shows as previously that the double cell $\eta=\left(F x \otimes_{h} \psi b^{\prime \prime}\right) \otimes_{v} f \xi$, in the right diagram above, has the same vertical composites with $\beta_{1}, \beta_{2}$.

(b) Assume that $f x$ is $V_{2}^{+}$-regular in $Y$. Given a pair of 2-cells $\alpha_{i}: a \rightarrow a_{i}: x \rightarrow x^{\prime}$ in the left diagram below, there exists in $Y$ a pair of double cells $\eta_{i}$ such that $f \alpha_{i} \otimes_{v} \eta_{i}=\eta$ (independent of $i$, see the right diagram)
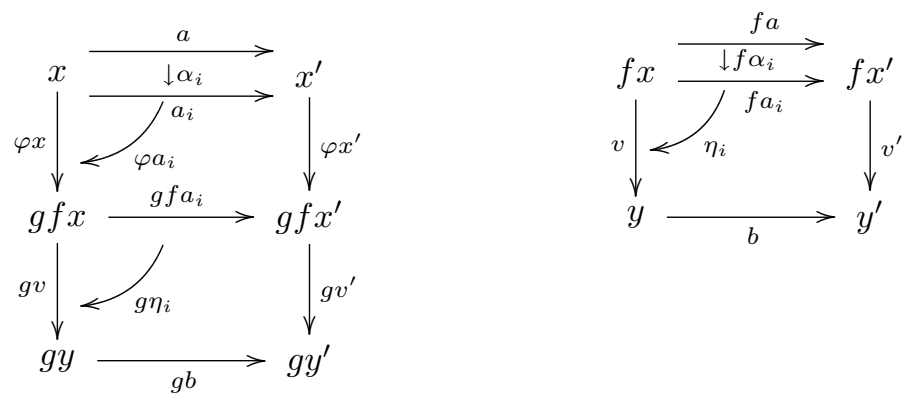

Then, in the left diagram above, the double cells $\xi_{i}=\varphi a_{i} \otimes_{v} g \eta_{i}$ solve our condition for the pair $\alpha_{i}$ :

$$
\alpha_{i} \otimes_{v} \varphi a_{i} \otimes_{v} g \eta_{i}=\varphi a \otimes_{v} g f \alpha_{i} \otimes_{v} g \eta_{i}=\varphi a \otimes_{v} g \eta .
$$

Finally, let $f x$ be $O_{2}^{+}$-regular in $Y$ and take, in $X$ (as in the left diagram below), three 2-cells $\alpha: a \rightarrow a^{\prime}: x \rightarrow x^{\prime}$ and $\alpha_{i}: a^{\prime} \rightarrow a^{\prime \prime}: x \rightarrow x^{\prime}$, so that $\alpha \otimes_{v} \alpha_{1}=$ $\alpha \otimes_{v} \alpha_{2}$; then there exists in $Y$ a 2-cell $\eta$ such that $f \alpha_{1} \otimes_{v} \eta=f \alpha_{2} \otimes_{v} \eta$. It follows, as previously, that in $X$ the double cell $\xi=\varphi a^{\prime \prime} \otimes_{v} g \eta$ has the same vertical composites with $\alpha_{1}, \alpha_{2}$
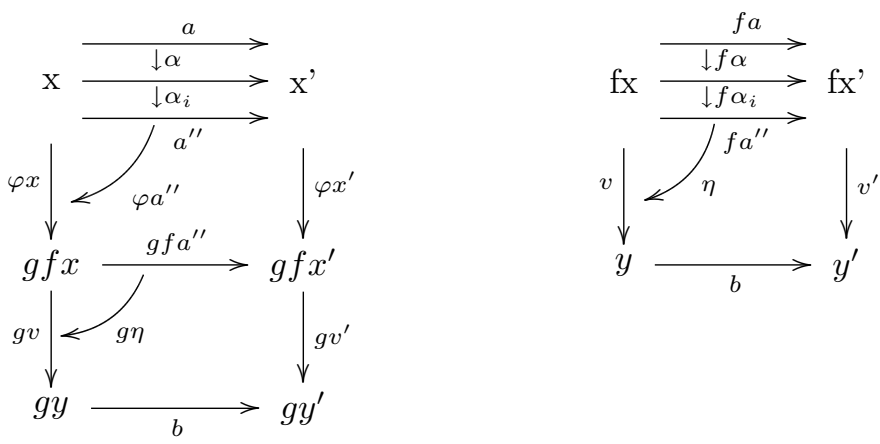

\section{The fundamental 2-category of a preordered space}

We define here the fundamental 2-category of a preordered space, and extend the construction to other settings. The index $\alpha$ takes values 0,1 (written,-+ in superscripts, as usual in cubical homotopical algebra). 


\subsection{The structure of the directed interval}

The directed interval $\uparrow \mathbf{I}$ is a lattice in pTop, with the following structural mappings: faces $\left(\delta^{\alpha}\right)$, degeneracy $(\varepsilon)$ and connections $\left(\gamma^{\alpha}\right.$, the binary operations of join and meet)

$$
\begin{gathered}
\{\star\} \frac{\delta^{-}}{\underset{\delta^{+}}{\rightleftarrows}} \uparrow \mathbf{I} \underset{\gamma^{+}}{\longleftarrow} \mathbf{I}^{2} \\
\delta^{-}(\star)=0, \quad \delta^{+}(\star)=1, \quad \gamma^{-}\left(t, t^{\prime}\right)=\max \left(t, t^{\prime}\right), \quad \gamma^{+}\left(t, t^{\prime}\right)=\min \left(t, t^{\prime}\right) .
\end{gathered}
$$

Actually, we are not interested in the complete axioms of lattices (e.g., the idempotence of the binary operations $\gamma^{ \pm}$, or their full absorption laws), but only in a part of them, corresponding to a cubical monoid in the sense of [10]: a set equipped with two structures of commutative monoid, so that the unit element of each operation is absorbent for the other. Formally, this means the following axioms, defining a cubical monoid in a monoidal category with tensor product $\times$ (with $\alpha, \beta= \pm$ and $\alpha \neq \beta$ )

$$
\begin{aligned}
& \varepsilon \cdot \delta^{\alpha}=1, \quad \varepsilon \cdot \gamma^{\alpha}=\varepsilon \cdot(\varepsilon \times \uparrow \mathbf{I})=\varepsilon \cdot(\uparrow \mathbf{I} \times \varepsilon) \quad(\text { degeneracy }), \\
& \gamma^{\alpha} \cdot\left(\gamma^{\alpha} \times \uparrow \mathbf{I}\right)=\gamma^{\alpha} \cdot\left(\uparrow \mathbf{I} \times \gamma^{\alpha}\right) \quad \text { (associativity), } \\
& \gamma^{\alpha} \cdot\left(\delta^{\alpha} \times \uparrow \mathbf{I}\right)=1=\gamma^{\alpha} \cdot\left(\uparrow \mathbf{I} \times \delta^{\alpha}\right) \quad \text { (unit), } \\
& \gamma^{\beta} \cdot\left(\delta^{\alpha} \times \uparrow \mathbf{I}\right)=\delta^{\alpha} \cdot \varepsilon=\gamma^{\beta} \cdot\left(\uparrow \mathbf{I} \times \delta^{\alpha}\right) \quad \text { (absorbent elements). }
\end{aligned}
$$

Higher faces, degeneracies and connections are constructed from the structural maps, via the monoidal structure, for $1 \leqslant i \leqslant n$ and $\alpha= \pm$

$$
\begin{aligned}
& \delta_{i}^{\alpha}=\uparrow \mathbf{I}^{i-1} \times \delta^{\alpha} \times \uparrow \mathbf{I}^{n-i}: \uparrow \mathbf{I}^{n-1} \longrightarrow \uparrow \mathbf{I}^{n}, \\
& \varepsilon_{i}=\uparrow \mathbf{I}^{i-1} \times \varepsilon \times \uparrow \mathbf{I}^{n-i}: \uparrow \mathbf{I}^{n} \longrightarrow \uparrow \mathbf{I}^{n-1}, \\
& \gamma_{i}^{\alpha}=\uparrow \mathbf{I}^{i-1} \times \gamma^{\alpha} \times \uparrow \mathbf{I}^{n-i}: \uparrow \mathbf{I}^{n+1} \longrightarrow \uparrow \mathbf{I}^{n},
\end{aligned}
$$

and the cocubical relations (for faces, degeneracy and connections) follow from this construction and the axioms above, in (75):

$$
\begin{array}{lll}
\delta_{j}^{\beta} \cdot \delta_{i}^{\alpha}=\delta_{i+1}^{\alpha} \cdot \delta_{j}^{\beta}(j \leqslant i), & \varepsilon_{i} \cdot \varepsilon_{j}=\varepsilon_{j} \cdot \varepsilon_{i+1}(j \leqslant i), & \\
\varepsilon_{j} \cdot \delta_{i}^{\alpha}=\delta_{i-1}^{\alpha} \cdot \varepsilon_{j}(j<i) & \text { or id }(j=i) & \text { or } \delta_{i}^{\alpha} \cdot \varepsilon_{j-1}(j>i), \\
\gamma_{j}^{\beta} \cdot \gamma_{i}^{\alpha}=\gamma_{i}^{\alpha} \cdot \gamma_{j+1}^{\beta}(j>i), & \gamma_{i}^{\alpha} \cdot \gamma_{i}^{\alpha}=\gamma_{i}^{\alpha} \cdot \gamma_{i+1}^{\alpha}, & \\
\varepsilon_{j} \cdot \gamma_{i}^{\alpha}=\gamma_{i-1}^{\alpha} \cdot \varepsilon_{j}(j<i) & \text { or } \varepsilon_{i} \cdot \varepsilon_{i}(j=i) & \text { or } \gamma_{i}^{\alpha} \cdot \varepsilon_{j+1} \quad(j>i), \\
\gamma_{j}^{\beta} \cdot \delta_{i}^{\alpha}=\delta_{i-1}^{\alpha} \cdot \gamma_{j}^{\beta}(j<i-1) & \text { or } \delta_{i}^{\alpha} \cdot \gamma_{j-1}^{\beta}(j>i), & \\
\gamma_{i}^{\alpha} \cdot \delta_{i}^{\alpha}=i d=\gamma_{i}^{\alpha} \cdot \delta_{i+1}^{\alpha}, & \gamma_{i}^{\beta} \cdot \delta_{i}^{\alpha}=\delta_{i}^{\alpha} \cdot \varepsilon_{i}=\gamma_{i}^{\beta} \cdot \delta_{i+1}^{\alpha}(\alpha \neq \beta) .
\end{array}
$$




\subsection{The cubical set of a preordered space}

Given a preordered space $X$, the previous structure of $\uparrow \mathbf{I}$ (and its powers, forming a cocubical object with connections in pTop) produces a cubical set with connections $P_{\star}(X)$

$$
\begin{array}{ll}
P_{n}(X)=\operatorname{pTop}\left(\uparrow \mathbf{I}^{n}, X\right), & \\
\partial_{i}^{\alpha}: P_{n}(X) \rightarrow P_{n-1}(X), & \partial_{i}^{\alpha}(a)=a \cdot \delta_{i}^{\alpha}: \uparrow \mathbf{I}^{n-1} \rightarrow X, \\
e_{i}: P_{n-1}(X) \rightarrow P_{n}(X), & e_{i}(a)=a \cdot \varepsilon_{i}: \uparrow \mathbf{I}^{n} \rightarrow X, \\
g_{i}^{\alpha}: P_{n}(X) \rightarrow P_{n+1}(X), & g_{i}^{\alpha}(a)=a \cdot \gamma_{i}^{\alpha}: \uparrow \mathbf{I}^{n+1} \rightarrow X,
\end{array}
$$

satisfying the cubical relations (dual to the cocubical ones, listed above).

\subsection{Moore paths and parallelepipeds}

Let us form the free cubical $\omega$-category $M_{\star}(X)$ on this cubical set $P_{\star}(X)$. A general item is a Moore parallelepiped, defined on a standard ordered $n$ parallelepiped (possibly degenerate)

$$
a: \prod_{j=1, \ldots, n} \uparrow\left[0, p_{j}\right] \rightarrow X \quad\left(p_{1}, \ldots, p_{n} \in \mathbf{N}\right) .
$$

These maps form the component $M_{n}(X)$. Faces and degeneracies are obvious (taking $\alpha=0,1$, still written,-+ in superscripts)

$$
\begin{array}{ll}
\partial_{i}^{\alpha}: M_{n}(X) \rightarrow M_{n-1}(X), \quad & \partial_{i}^{\alpha}(a)\left(t_{1}, \ldots, t_{n-1}\right)=a\left(t_{1}, \ldots, \alpha p_{i}, \ldots, t_{n-1}\right), \\
e_{i}: M_{n-1}(X) \rightarrow M_{n}(X), & e_{i}(a)\left(t_{1}, \ldots, t_{n}\right)=a\left(t_{1}, \ldots, \hat{t}_{i}, \ldots, t_{n}\right)
\end{array}
$$

and defined, respectively, on the standard parallelepipeds given by the natural numbers

$$
\left(p_{1}, \ldots, \hat{p}_{i}, \ldots, p_{n}\right) \quad\left(p_{1}, \ldots, p_{i-1}, 0, \ldots, p_{n-1}\right) .
$$

The $i$-composition of two Moore parallelepipeds $a, b$ with $\partial_{i}^{+}(a)=\partial_{i}^{-}(b)$ (defined on $\prod \uparrow\left[0, p_{j}\right]$ and $\prod \uparrow\left[0, q_{j}\right]$, respectively) is also obvious

$$
\left(a \star_{i} b\right)\left(t_{1}, \ldots, t_{n}\right)= \begin{cases}a\left(t_{1}, \ldots, t_{i}, \ldots, t_{n}\right), & 0 \leqslant t_{i} \leqslant p_{i} \\ b\left(t_{1}, \ldots, t_{i}-p_{i}, \ldots, t_{n}\right), & p_{i} \leqslant t_{i} \leqslant p_{i}+q_{i},\end{cases}
$$

with identities given by the degeneracies $e_{i}$.

This cubical set has 'pre-connections', whose 'degenerate' faces are constant (instead of being actual identities)

$$
g_{i}^{\alpha}: M_{n}(X) \rightarrow M_{n+1}(X), g_{i}^{\alpha}(a)\left(t_{1}, \ldots, t_{n+1}\right)=a\left(t_{1}, \ldots, \gamma^{\alpha}\left(t_{i}, t_{i+1}\right), \ldots, t_{n+1}\right),
$$

where $g_{i}^{\alpha}(a)$ is defined on $\uparrow\left[0, p_{1}\right] \times \cdots \times \uparrow\left[0, p_{i}\right]^{2} \times \cdots \times \uparrow\left[0, p_{n}\right]$. 
Truncating $M_{\star}(X)$ at dimension 2, we get a cubical 2-category (i.e., an edgesymmetric double category, where horizontal and vertical maps coincide) with pre-connections

$$
M_{0}(X) \stackrel{\partial^{\alpha}}{\longleftarrow} M_{1}(X) \stackrel{\longleftrightarrow \ldots \ldots}{\leftrightarrows} M_{2}(X) .
$$

Further, replacing $M_{2}(X)$ with $N_{2}(X)=M_{2}(X) / \simeq$ (modulo homotopy with fixed boundary) and leaving $N_{i}(X)=M_{i}(X)$ for $i=0,1$, we have again a cubical 2-category with pre-connections.

\subsection{Congruences}

Let $C$ be a cubical 2-category. A congruence $R=\left(R_{0}, R_{1}, R_{2}\right)$ in $C$ will be a triple of equivalence relations, one in each component $C_{0}, C_{1}, C_{2}$ (of objects, maps and cells) of $C$, so that these relations are:

(i) consistent with faces and degeneracies,

(ii) consistent with each $i$-composition law, in the following sense: if $a, b \in C_{n}$ and their faces $\partial_{i}^{+} a$ and $\partial_{i}^{-} b$ are equivalent (modulo $R_{n-1}$ ), then:

- there exist $a^{\prime}, b^{\prime}$ equivalent to $a, b$ (modulo $R_{n}$ ) which are $i$-consecutive, i.e., $\partial_{i}^{+} a^{\prime}=\partial_{i}^{-} b^{\prime}$,

- if $a^{\prime \prime}, b^{\prime \prime}$ are also so, then the $i$-composites $a^{\prime} \star_{i} b^{\prime}$ and $a^{\prime \prime} \star_{i} b^{\prime \prime}$ are $R_{n}$-equivalent.

Plainly, the quotient 2-truncated cubical set $C / R$ (with components $C_{n} / R_{n}$, the induced faces and degeneracies) inherits well-defined $i$-composition laws, which make it into a cubical 2-category: the quotient of the cubical 2-category $C$ modulo $R$.

\subsection{The fundamental 2-category}

Now, starting from the cubical 2-category with pre-connections $N_{\star}(X)$ defined at the end of 5.3, we form a double category with connections

$$
D_{\star}(X)=N_{\star}(X) / R,
$$

identifying 'pre-identities' (cubes which are constant in some direction) with identities.

In other words, we define the congruence $R(5.4)$ of $N_{\star}(X)$ as follows. $R_{0}$ is the equality of $N_{0}(X)=|X| ; R_{1}$ is the least equivalence relation of $N_{1}(X)=M_{1}(X)$ closed under concatenation which identifies every constant path $a: \uparrow[0, p] \rightarrow X$ with its faces $\{0\} \rightarrow X ; R_{2}$ is the least equivalence relation of $N_{2}(X)$, closed under 1 - and 2-concatenation, which identifies the class of any 'rectangle' $a: \uparrow[0, p] \times \uparrow[0, q]$, constant in direction $i$, with $e_{i} \partial_{i}^{-} a$.

The conditions 5.4 (i) and (ii) are satisfied: the only point which needs some explanation is the first part of (ii), for $n=2$. Assume that the 2-dimensional items $a, b: \uparrow[0, p] \times \uparrow[0, q] \rightarrow X$ have $R_{1}$-equivalent faces $\partial_{2}^{+}[a]=\partial_{2}^{+} a, \partial_{2}^{-}[b]=\partial_{2}^{-} b$. Whenever we modify the path $c=\partial_{2}^{+} a: \uparrow[0, p] \rightarrow X$ with the insertion of a constant 
piece at $p^{\prime}\left(0 \leqslant p^{\prime} \leqslant p\right)$, of length $m \in N$

$$
c^{\prime}(t)= \begin{cases}c(t), & 0 \leqslant t \leqslant p^{\prime}, \\ c\left(p^{\prime}\right), & p^{\prime} \leqslant t \leqslant p^{\prime}+m, \\ c(t-m), & p^{\prime}+m \leqslant t \leqslant p+m,\end{cases}
$$

this modification can be accompanied with a similar modification on $a$ (in the first variable), obtaining a map

$$
a^{\prime}: \uparrow[0, p+m] \times \uparrow[0, q] \rightarrow X, \quad \partial_{2}^{+} a^{\prime}=c^{\prime} .
$$

Continuing this way, we end with replacing $a, b$ with equivalent items $\bar{a}, \bar{b}$ having $\partial_{2}^{+} \bar{a}=\partial_{2}^{+} \bar{b}$.

Now, the fundamental cubical 2-category of $X$ is defined as the quotient $N_{\star}(X) / R$. The fundamental 2-category is obtained in the usual way, restricting double cells to those whose faces in direction 2 (for instance) are trivial. We have thus a functor

$$
\uparrow \Pi_{2}: \mathrm{p} \text { Top } \rightarrow 2-\text { Cat. }
$$

Furthermore, a directed homotopy $\varphi: f \rightarrow g: X \rightarrow Y$ induces a lax natural transformation $\varphi_{\star}: f_{\star} \rightarrow g_{\star}: \uparrow \Pi_{2}(X) \rightarrow \uparrow \Pi_{2}(Y)$, sending the point $x \in X$ to the $Y$-arrow $\varphi_{\star}(x): f_{\star}(x) \rightarrow g_{\star}(x)$ determined by the path $\varphi(x,-): f x \rightarrow f x^{\prime}$ and the arrow $[a]: x \rightarrow x^{\prime}$ (determined by a path in $X$ ) to the 2-cell $\varphi_{\star}[a]$ corresponding to the double homotopy $\varphi \circ(a \times \uparrow \mathbf{I}): \uparrow \mathbf{I} \times \uparrow \mathbf{I} \rightarrow Y$

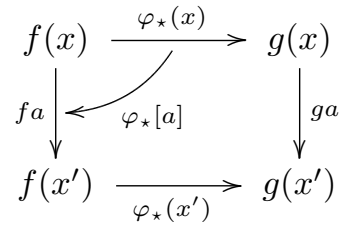

\subsection{Other directed structures}

In a preordered space, every loop is reversible (as already remarked in 1.1); therefore, this setting has no 'directed circle' or 'directed torus'.

We briefly recall more complex directed structures, which allow for non-reversible loops. All of them have a directed interval $\uparrow \mathbf{I}$ with the structure considered above, so that all the previous constructions can be easily extended. Furthermore, all of them have a reflection $X \mapsto X^{\mathrm{op}}$ extending the preorder-reversion of pTop.

First, one could extend pTop by some local notion of ordering. The simplest way is perhaps to consider spaces equipped with a relation $\prec$ which is reflexive and locally transitive: every point has some neighbourhood on which the relation is transitive ([11], 1.4); similar, stronger properties have been frequently used in the theory of concurrency. But a relevant internal drawback appears, which makes this setting inadequate for directed homotopy and homology: mapping cones and suspension are lacking. Indeed, a locally preordered space cannot have a 'point-like vortex' (where all neighbourhoods of a point contain some non-reversible loop), whence it cannot realise the cone of the directed circle (as proved in detail in [11], 4.6). 


\subsection{Spaces with distinguished paths}

A sufficiently general, well-behaved setting has been studied in [11].

A $d$-space $X=(X, d X)$ is a topological space equipped with a set $d X$ of (continuous) maps $a: \mathbf{I} \rightarrow X$; these maps, called distinguished paths or directed paths or d-paths, must contain all constant paths and be closed under concatenation and increasing reparametrisation (i.e., pre-composing with a weakly increasing continuous mapping $\mathbf{I} \rightarrow \mathbf{I})$.

A $d$-map $f: X \rightarrow Y$ (or map of d-spaces) is a continuous mapping between d-spaces which preserves the directed paths: if $a \in d X$, then $f a \in d Y$.

The category of d-spaces is written as dTop. It has all limits and colimits, constructed as in Top and equipped with the initial or final d-structure for the structural maps; for instance a path $\mathbf{I} \rightarrow \prod X_{j}$ is directed if and only if all its components $\mathbf{I} \rightarrow X_{j}$ are so. The forgetful functor $U: \mathrm{d}$ Top $\rightarrow$ Top preserves thus all limits and colimits; a topological space is generally viewed as a d-space by its natural structure, where all (continuous) paths are directed (via the right adjoint to $U$ ).

Reversing d-paths, by the involution $r(t)=1-t$, yields the reflected, or opposite, d-space $R X=X^{\mathrm{op}}$, where $a \in d\left(X^{\mathrm{op}}\right)$ if and only if $a^{\mathrm{op}}=a r \in d X$.

The standard d-interval $\uparrow \mathbf{I}=\uparrow[0,1]$ has directed paths given by the (weakly) increasing maps $\mathbf{I} \rightarrow \mathbf{I}$. The standard d-circle $\uparrow \mathbf{S}^{1}=\uparrow \mathbf{I} / \partial \mathbf{I}$ has the (obvious) quotient d-structure, where paths have to follow a precise orientation (but note that the directed structure $\uparrow \mathbf{S}^{1} \times \uparrow \mathbf{S}^{1}$ on the torus has nothing to do with orientation).

An alternative setting, inequilogical spaces, introduced in [13] as a directed version of Dana Scott's equilogical spaces $[\mathbf{1 8}, \mathbf{1}]$, could also be used - but would require a more complicated procedure to concatenate paths and homotopies (cf. $[\mathbf{1 3}])$.

\subsection{Geometrical aspects of the congruence}

Defining higher fundamental categories $\uparrow \Pi_{n}(X)$ with $n>2$ is even more complicated. In [12], we thought that the problem might be solved, identifying Moore parallelepipeds modulo delays in each variable. However, this is not consistent with concatenations.

For instance, consider two cubes $a, b \in C_{2}$ with a common degenerate face $\partial_{1}^{+} a=\partial_{1}^{-} b=e_{1}(x)$ (represented below as a double line). Then, their concatenation $c=a \star_{1} b$ is $R_{2}$-equivalent to the pasting $c^{\prime}=\left(a \star_{2} a^{\prime}\right) \star_{1}\left(b^{\prime} \star_{2} b\right)$, also represented below, where $a^{\prime}$ and $b^{\prime}$ are constant in direction 2
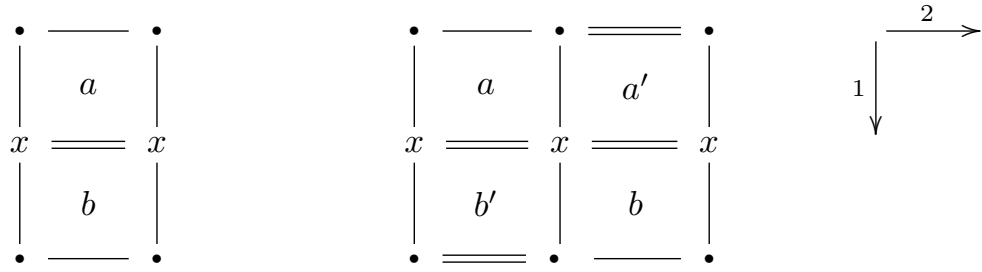
Now, $a \equiv_{2}\left(a \star_{2} a^{\prime}\right)$ and $b \equiv_{2}\left(b^{\prime} \star_{2} b\right)$ (under equivalence modulo delays), but $c$ and $c^{\prime}$ are not equivalent modulo delays, in general. Note also that such a modification of $c$ into $c^{\prime}$, by a sort of 'generalised delay', requires a constraint on the common face $\partial_{1}^{+} a=\partial_{1}^{-} b$ (i.e., being degenerate). Thus, a global description of $R_{n}$ (as 'attempted' in [12]) should be complicated.

\section{Modelling a fundamental 2-category}

We study the fundamental 2-category of the 'hollow cube' $X \subset \uparrow[0,1]^{3}$ (see (8)), starting with the construction of a future 2-equivalent model.

The canonical basis of the real vector space $\mathbf{R}^{3}$ is denoted $e_{1}=(1,0,0)$, etc. By abuse of notation, the 2-cell $[\varphi]: a \rightarrow b$ associated to a 2-homotopy $\varphi: a \rightarrow b$ is generally written as $\varphi$; the context tells the difference.

\subsection{Bi-affine maps}

We shall need to consider biaffine maps $\alpha: \mathbf{I}^{2} \rightarrow \mathbf{R}^{n}$, i.e., mappings which are affine in both variables (on the standard square). Such a mapping determines a 4-tuple of points, the images of the four vertices of the standard square, which will be called the vertices of the map

$$
p_{i j}=\alpha(i, j) \quad(i, j) \in\{0,1\}^{2} .
$$

The correspondence is bijective: given an arbitrary 4 -tuple $\left(p_{i j}\right)$ of points in $\mathbf{R}^{n}$, the biaffine map is reconstructed by the following formula:

$$
\alpha\left(t_{1}, t_{2}\right)=\left(1-t_{1}\right)\left(1-t_{2}\right) p_{00}+t_{1}\left(1-t_{2}\right) p_{10}+\left(1-t_{1}\right) t_{2} p_{01}+t_{1} t_{2} p_{11} .
$$

In this way, we get a map $\alpha: \uparrow \mathbf{I}^{2} \rightarrow \uparrow \mathbf{R}^{n}$ (preserving the canonical orders) if and only if, in $\mathbf{R}^{n}$ :

$$
p_{00} \leqslant p_{10} \leqslant p_{11}, \quad p_{00} \leqslant p_{01} \leqslant p_{11},
$$

which amounts to saying that the mapping $p:\{0,1\}^{2} \rightarrow \mathbf{R}^{n}$ is order-preserving.

\subsection{Studying the singularity}

Now, the singularity in the 'hollow cube' $X \subset \uparrow[0,1]^{3}$ (defined in (8)) is made evident by the existence of two different cells $\alpha, \beta: a \rightarrow b: p_{0} \rightarrow q_{0}$ (with values in the 'internal boundary'), for suitable paths $a, b$, from $p_{0}=(1 / 3,1 / 3,1 / 3)$ to $q_{0}=(2 / 3,2 / 3,2 / 3)$. (These cells also show that $p_{0}$ is a $V_{2}^{+}$-branching point of the fundamental 2-category, cf. 4.4.)

Let us construct an example. The images of the 2-homotopies $\alpha, \beta$, in the picture below, cover the faces of the cubic hole $] 1 / 3,2 / 3\left[^{3}\right.$, forming its upper-left and its 
lower-right half, separated by the paths $a, b$

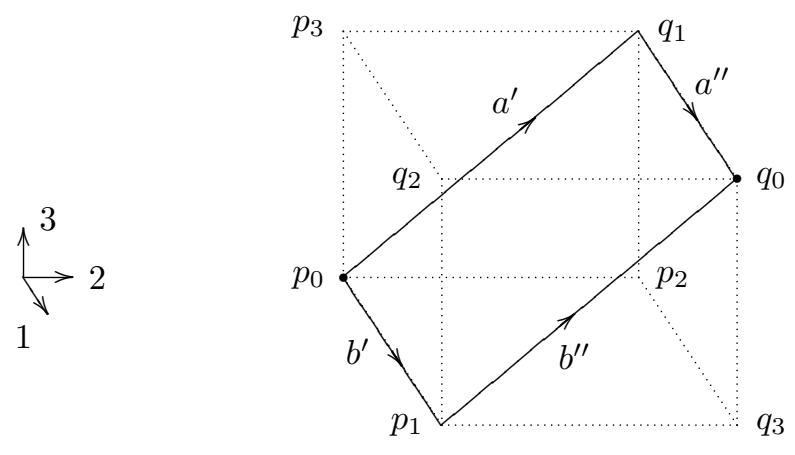

$$
p_{i}=p_{0}+e_{i} / 3, \quad q_{i}=q_{0}-e_{i} / 3, \quad a=a^{\prime} \star a^{\prime \prime}, \quad b=b^{\prime} \star b^{\prime \prime} .
$$

(Recall that the vectors $e_{1}, e_{2}, e_{3}$ form the canonical basis of the vector space $\mathbf{R}^{3}$.) More precisely, the 2-homotopies $\alpha, \beta: a \rightarrow b: p_{0} \rightarrow q_{0}$ are obtained from the double homotopies $\alpha^{\prime}, \beta^{\prime}$ represented and described below, in the obvious way (i.e., using connections; cf. 5.1)
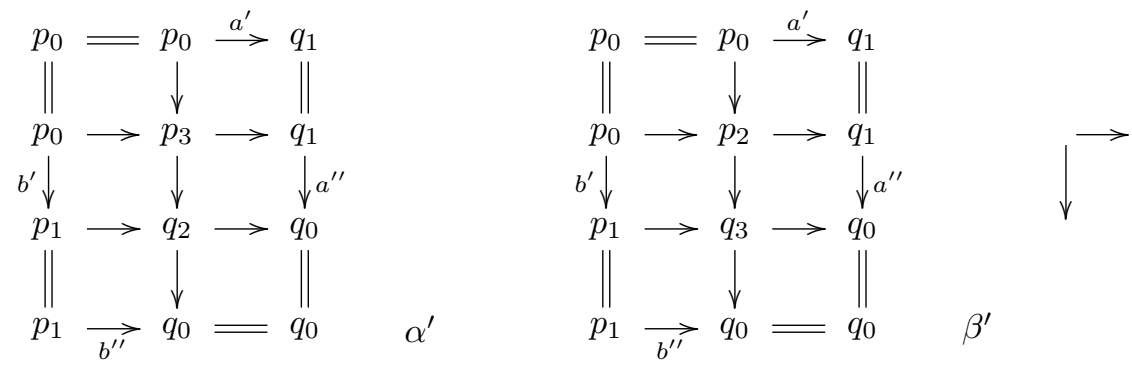

The double homotopy $\alpha^{\prime}$ is piecewise biaffine, made up of the pasting of six biaffine maps $\uparrow \mathbf{I}^{2} \rightarrow X$, each of them determined by its four vertices in $X$ (as specified in the left diagram); similarly for $\beta^{\prime}$ (in the right diagram). The mappings $\alpha^{\prime}, \beta^{\prime}$ are constant on the double lines. (One could further draw the connections yielding $\alpha$ and $\beta$, but this would complicate the picture rather than making it clearer.)

Let us rename the paths $a, b$ as $a_{1}, b_{1}$ (they go through $q_{1}$ and $p_{1}$, respectively) and the 2-homotopies $\alpha, \beta$ as $\alpha_{12}$ (through $q_{2}$ ) and $\alpha_{13}$ (through $q_{3}$ ). By permutation of coordinates, we get two other similar pairs of cells (each linking a pair of paths); altogether, we have six 2-cells

$$
\alpha_{i j}: a_{i} \rightarrow b_{i}: p_{0} \rightarrow q_{0} \quad(i, j=1,2,3 ; i \neq j) .
$$

Actually, we shall need a slightly more general construct, where we replace $q_{0}$ with any $q_{0}^{\prime} \geqslant q_{0}$ (in $X$ ). The picture below represents the modified $\alpha_{12}, \alpha_{13}: a_{1} \rightarrow b_{1}$, which (now) go from $p_{0}$ to $q_{0}^{\prime}$ 


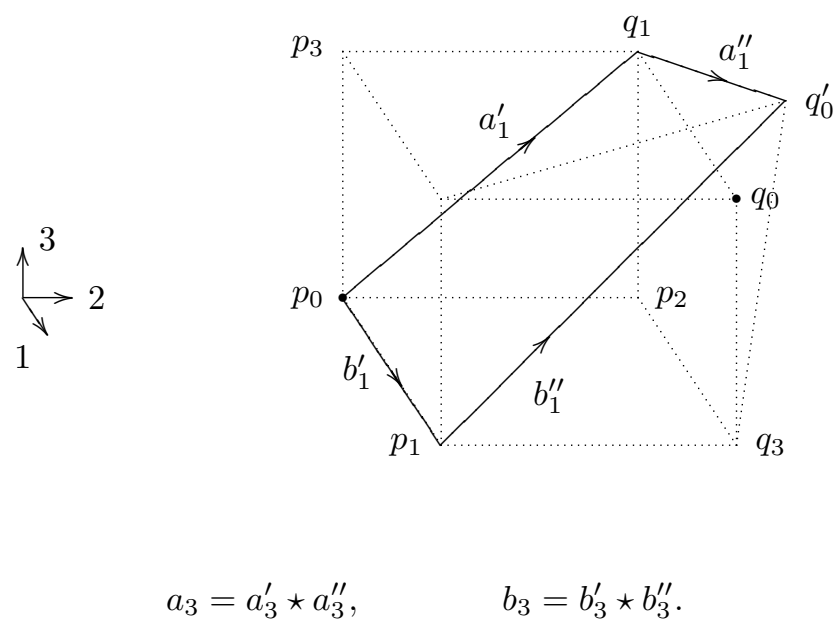

\subsection{The future model}

We define now a sub-2-category $C^{+}$of $C_{2}=\uparrow \Pi_{2}(X)$ which will be proved to be future 2-equivalent to $C_{2}$ (and corresponds to the future model $F$ of the 'hollow square', in (5)).

$C^{+}$has eight objects, forming a set $V^{+}$: the seven vertices of the cubic hole different from $q_{0}=(2 / 3,2 / 3,2 / 3)$, plus $q_{0}^{\prime}$, the maximum of $X$

$$
\begin{aligned}
& p_{0}=(1 / 3,1 / 3,1 / 3), \quad q_{0}^{\prime}=(1,1,1), \\
& p_{i}=p_{0}+e_{i} / 3, \quad q_{i}=q_{0}-e_{i} / 3 \quad(i=1,2,3) \text {. }
\end{aligned}
$$

After identities, it has the following 12 affine paths (determined by their vertices)

$$
\begin{aligned}
& a_{i}^{\prime}: p_{0} \rightarrow q_{i}, \quad a_{i}^{\prime \prime}: q_{i} \rightarrow q_{0}^{\prime}, \\
& b_{i}^{\prime}: p_{0} \rightarrow p_{i}, \quad b_{i}^{\prime \prime}: p_{i} \rightarrow q_{0}^{\prime} \quad(i=1,2,3),
\end{aligned}
$$

and their six composites, piecewise affine (more precisely, their equivalence classes modulo delays, cf. 5.5)

$$
a_{i}=a_{i}^{\prime} \star a_{i}^{\prime \prime}: p_{0} \rightarrow q_{0}^{\prime}, \quad b_{i}=b_{i}^{\prime} \star b_{i}^{\prime \prime}: p_{0} \rightarrow q_{0}^{\prime} .
$$

Finally, $C^{+}$is locally full in $C_{2}$, which means that it has 12 non-trivial cells, precisely the six $\alpha_{i j}$ analytically defined in 6.2 and six other cells $\gamma_{i j}$ determined by their boundary

$$
\alpha_{i j}: a_{i} \rightarrow b_{i}: p_{0} \rightarrow q_{0}^{\prime}, \quad \gamma_{i j}: b_{i} \rightarrow a_{j}: p_{0} \rightarrow q_{0}^{\prime} \quad(i, j=1,2,3 ; i \neq j) .
$$

The figure below shows the 2-homotopy $\gamma_{12}: b_{1} \rightarrow a_{2}$ 

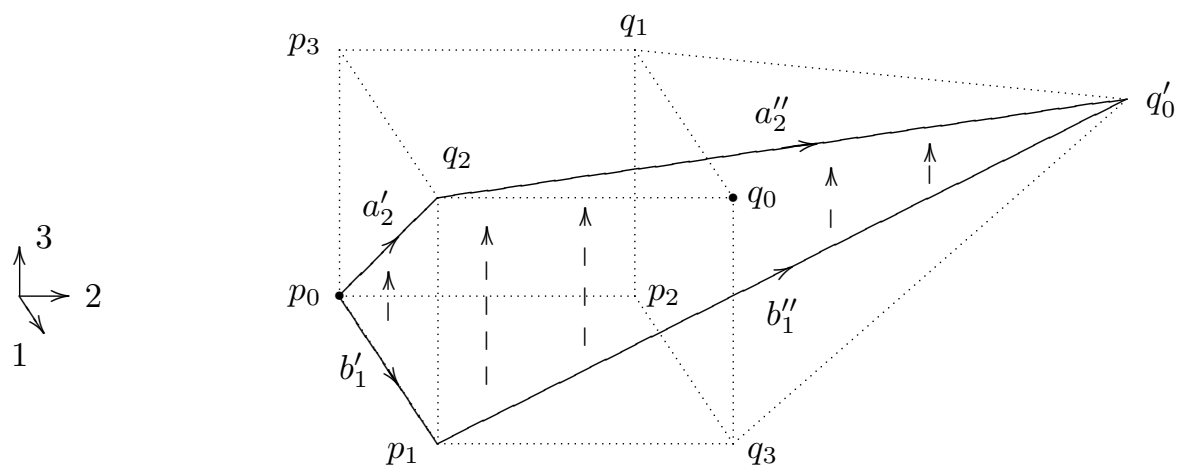

$$
a_{2}=a_{2}^{\prime} \star a_{2}^{\prime \prime}, \quad b_{1}=b_{1}^{\prime} \star b_{1}^{\prime \prime}
$$

\subsection{The retraction}

Let us consider a partition of the space $X$ into eight zones: the points which are below precisely one vertex of $V^{+}$

$$
\begin{aligned}
& P_{0}=\downarrow p_{0}, \quad P_{i}=\downarrow p_{i} \backslash \downarrow p_{0} \quad \text { (four cubes), } \\
& Q_{1}=\downarrow q_{1} \backslash\left(\downarrow p_{2} \cup \downarrow p_{3}\right), \quad Q_{2}=\ldots, \quad Q_{3}=\ldots \quad \text { (three cubes), } \\
& Q=X \backslash\left(\downarrow q_{1} \cup \downarrow q_{2} \cup \downarrow q_{3}\right) \text {. }
\end{aligned}
$$

It will be useful to note that, if $Y$ is any of these eight zones and $y \in Y$, the subspace $Y \cap \uparrow y$ is compact. As a consequence, a path $a: \uparrow \mathbf{I} \rightarrow X$ which meets $Y$ has a last moment in it, $t_{0} \leqslant 1$

$$
t_{0}=\max \{t \in \mathbf{I} \mid a(t) \in Y\}
$$

if $t_{0}<1$, the path gets out of $Y$ and never gets back.

Now, the 2-functor $p: C_{2} \rightarrow C^{+}$is defined as follows. It sends each point $x \in X$ to

$$
p(x)=\min \left\{v \in V^{+} \mid x \leqslant v\right\} .
$$

Let $a: x^{\prime} \rightarrow x^{\prime \prime}$. Then there is a unique path $p(a): p\left(x^{\prime}\right) \rightarrow p\left(x^{\prime \prime}\right)$ in $C^{+}$, excepting the case when $p\left(x^{\prime}\right)=p_{0}$ and $p\left(x^{\prime \prime}\right)=q_{0}^{\prime}$; in this case $p(a)$ is defined to be:

$$
p(a)= \begin{cases}a_{i}=a_{i}^{\prime} \star a_{i}^{\prime \prime}, & \text { if } a, \text { when leaving } P_{0}, \text { enters } Q_{i}, \\ b_{i}=b_{i}^{\prime} \star b_{i}^{\prime \prime}, & \text { if } a, \text { when leaving } P_{0}, \text { enters } P_{i} .\end{cases}
$$

Let $\alpha: a \rightarrow b: x^{\prime} \rightarrow x^{\prime \prime}$. Then, there is a unique cell $p(\alpha): p(a) \rightarrow p(b)$ in $C^{+}$, unless $p(a)=a_{i}$ and $p(b)=b_{i}$ (with the same index $i$ ); in this case, there are two 
such cells $\alpha_{i j}$, and we define

$$
p(\alpha)=\alpha_{i j}: a_{i} \rightarrow b_{i}, \quad \text { if } \alpha \text { meets } Q_{j}(j \neq i) .
$$

\subsection{The future equivalence}

The inclusion $f: C^{+} \rightarrow C_{2}$ forms, with $p$, a future 2-retract

$$
f: C^{+} \rightleftarrows C_{2}: p, \quad \quad p f=1_{C^{+}}, \quad \eta: 1_{C_{2}} \rightarrow f p,
$$

where the lax natural transformation $\eta$ is defined as follows.

For $x \in X$, let $\eta x: x \rightarrow p(x)$ be the affine path with these endpoints (which are contained in the down-set $\downarrow p(x))$. For $a: x^{\prime} \rightarrow x^{\prime \prime}$, let $\eta a: \eta x^{\prime \prime} \cdot a \rightarrow p a \cdot \eta x^{\prime}$ be the 2 -cell associated to the biaffine double cell $\hat{\eta} a$ (as in the left diagram below)
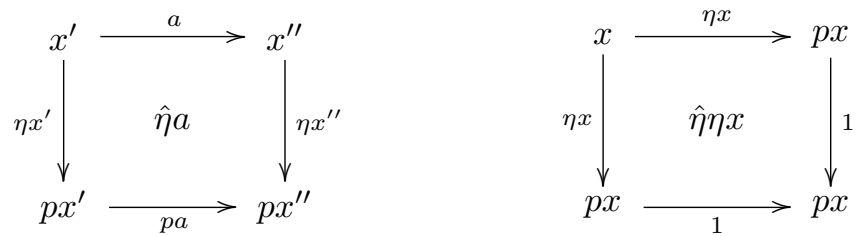

Plainly, $p \eta=1_{p}$ and $\eta f=1_{f}$; finally, the coherence condition $(35)\left(\eta \star \eta=1_{\eta}\right)$ is satisfied, since $\hat{\eta} \eta x$ (see the right diagram above) is the lower connection on the path $\eta x$.

\subsection{The past model}

Symmetrically, we have a past 2-retract $C^{-}$of $C_{2}$, whose set of objects $V^{-}$ consists of the seven vertices of the cubic hole different from $p_{0}=(1 / 3,1 / 3,1 / 3)$, together with the minimum $p_{0}^{\prime}$ of $X$

$$
\begin{array}{lll}
p_{0}^{\prime}=(0,0,0), & q_{0}=(2 / 3,2 / 3,2 / 3), & \\
p_{i}=p_{0}+e_{i} / 3, & q_{i}=q_{0}-e_{i} / 3
\end{array} \quad(i=1,2,3) .
$$

\subsection{A global 2-model}

Finally, the pf-2-presentation of $C_{2}$ by the future 2-retract $C^{+}$and the past 2-retract $C^{-}$generates an injective 2-model $E(4.3)$, on ten objects

$$
V=V^{-} \cup V^{+}=\left\{p_{0}^{\prime}, p_{i}, q_{i}, q_{0}^{\prime}\right\} \quad(i=0,1,2,3) .
$$

\section{Appendix: the calculus of lax natural transformations and modifications}

We end with reviewing the various compositions of the notions recalled in Section 2. Again, we always consider strict 2-functors between 2-categories.

\subsection{Composing transformations}

(a) Given two lax natural transformations $\varphi: f \rightarrow g, \psi: g \rightarrow h$ between 2-functors $X \rightarrow Y$, the (main) composition $\psi \varphi: f \rightarrow h$ has components produced by the 
vertical pasting of double cells in $Y$, and is therefore strictly associative, with strict identities

$$
(\psi \varphi) x=\psi x \cdot \varphi x, \quad(\psi \varphi) a=\varphi a \otimes_{v} \psi a,
$$

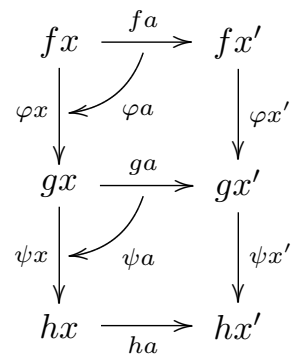

It is easy to see that $\varphi: f \rightarrow g$ is invertible (for this composition) if and only if all its components $\varphi x: f x \rightarrow g x$ and $\varphi a: \varphi x^{\prime} \cdot f a \rightarrow g a \cdot \varphi x$ are invertible in $Y$. Then, one defines the inverse $\psi: g \rightarrow f$ letting $\psi x=(\varphi x)^{-1}$ and $\psi a=\psi x^{\prime} \cdot(\varphi a)^{-1} \cdot \psi x$.

(b) The whisker composition $k \varphi h: k f h \rightarrow k g h$ (for $h: X^{\prime} \rightarrow X, k: Y \rightarrow Y^{\prime}$ ) has the obvious components, produced by evaluation

$$
(k \varphi h) x=k \varphi h(x), \quad(k \varphi h) a=k \varphi h(a), \quad\left(a: x \rightarrow x^{\prime} \text { in } X^{\prime}\right),
$$

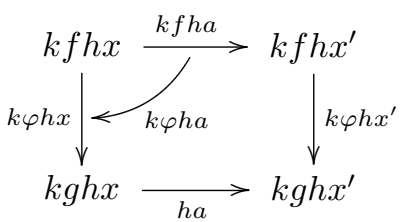

We have thus a sesquicategory Lnt of 2-categories, 2-functors and lax natural transformations, which is not a 2-category: the reduced interchange axiom (2.1) does not hold (see also 7.3).

\subsection{Composing modifications}

(a) First, we have an obvious whisker composition of modifications and 2-functors (for $M: \varphi \rightarrow \psi: f \rightarrow g: X \rightarrow Y, h: X^{\prime} \rightarrow X$ and $k: Y \rightarrow Y^{\prime}$ )

$$
k M h: k \varphi h \rightarrow k \psi h: k f h \rightarrow k g h: X^{\prime} \rightarrow Y^{\prime} .
$$

But, given the 2-categories $X, Y$, the important construct is the 2-category $\operatorname{Lnt}(X, Y)$ of 2 -functors $X \rightarrow Y$, their lax natural transformations and modifications. Indeed, modifications have a main composition

$$
\begin{array}{ll}
M: \varphi \rightarrow \psi: f \rightarrow g: X \rightarrow Y, & N: \psi \rightarrow \xi: f \rightarrow g: X \rightarrow Y, \\
N M: \varphi \rightarrow \xi, & (N M) x=N x \cdot M x: \varphi x \rightarrow \psi x \rightarrow \xi x,
\end{array}
$$


Homology, Homotopy and Applications, vol. 8(1), 2006

which is strictly associative, with obvious identities $1_{\varphi}$. (For this law, the modification $M$ is invertible if and only if all its component cells $M x: \varphi x \rightarrow \psi x$ are invertible in $Y$, and then $M^{-1}(x)=(M x)^{-1}$.)

(b) Moreover, we have an obvious whisker composition of the modification $M$ with lax natural transformations $\lambda: f^{\prime} \rightarrow f$ and $\mu: g \rightarrow g^{\prime}$

$$
\begin{aligned}
& \mu M \lambda: \mu \varphi \lambda \rightarrow \mu \psi \lambda: f^{\prime} \rightarrow g^{\prime}: X \rightarrow Y, \\
& (\mu M \lambda) x=\mu x \cdot M x \cdot \lambda x: \mu x \cdot \varphi x \cdot \lambda x \rightarrow \mu x \cdot \psi x \cdot \lambda x: f^{\prime} x \rightarrow g^{\prime} x, \\
& f^{\prime} x \stackrel{\lambda x}{\longrightarrow} f x \stackrel{\frac{\varphi x}{\downarrow M x}}{\frac{\downarrow M x}{\longrightarrow}} g x \stackrel{\mu x}{\longrightarrow} g^{\prime} x .
\end{aligned}
$$

The reduced interchange axiom holds (as soon as it holds in $Y$ )

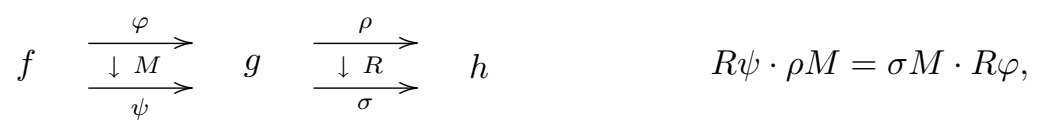

since its verification on a general component depends on the same property in $Y$ :

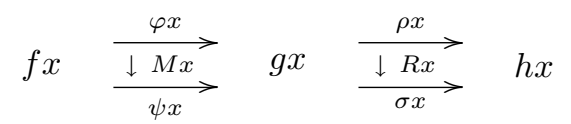

\subsection{Higher compositions}

Coming back to Lnt, we already observed that this sesquicategory is not a 2 -category. But one can define a graded composition of lax natural transformations $\rho \star \varphi$, as a modification. In fact, every object $x$ is taken to an arrow $\varphi x: f x \rightarrow g x$ of $Y$, and then to a cell $\rho(\varphi x)$ of $Z$

$$
X \underset{g}{\stackrel{f \varphi}{\longrightarrow}} Y \underset{s}{\stackrel{r \rho}{\longrightarrow}} Z
$$

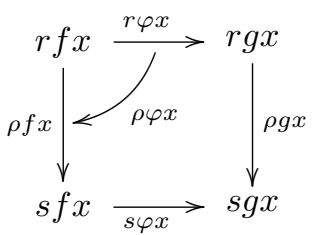

$\rho \star \varphi: \rho g \cdot r \varphi \rightarrow s \varphi \cdot \rho f, \quad(\rho \star \varphi) x=\rho(\varphi x): \rho g x \cdot r \varphi x \rightarrow s \varphi x \cdot \rho f x: r f x \rightarrow s g x$. 
To verify the axiom (mdf), take a map $a: x \rightarrow x^{\prime}$ in $X$. Then:

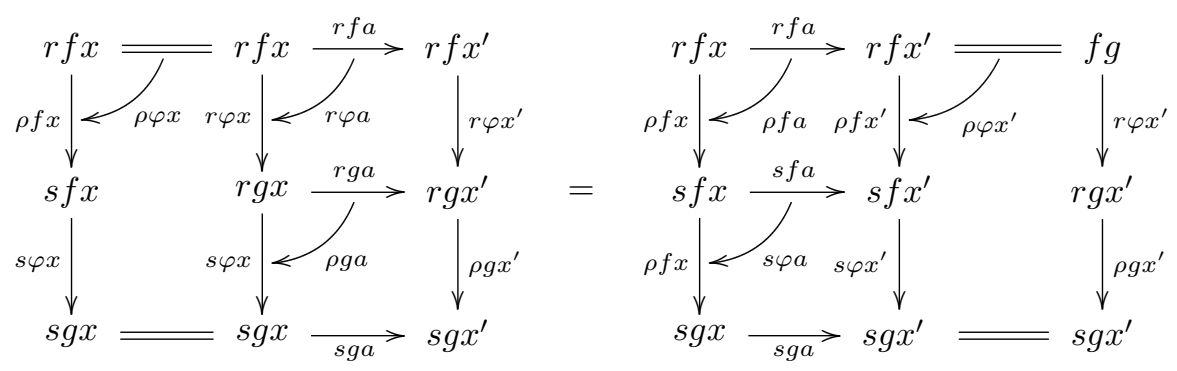

In fact, applying (lnt.2) to the lax natural transformation $\rho$, the left and the right pasting in (119) give, respectively

$$
r \varphi a \otimes_{v} \rho(g a \cdot \varphi x), \quad \rho\left(\varphi x^{\prime} \cdot f a\right) \otimes_{v} s \varphi a,
$$

and these results coincide, by (lnt.3), applied to $\rho$ and the cell $\varphi a: \varphi x^{\prime} \cdot f a \rightarrow$ $g a \cdot \varphi x$.

Then, in the following situation

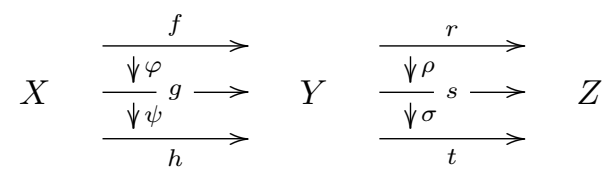

we have a pasting of modifications in $\operatorname{Lnt}(X, Z)$, which coincides with $(\psi \varphi) \star(\sigma \rho)$

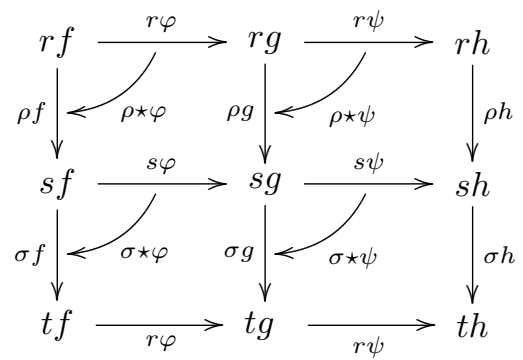

\subsection{Higher compositions, II}

One can also define a higher whisker composition of lax natural transformations and modifications.

(a) First, given a modification $M: \varphi \rightarrow \psi$ and a lax natural transformation $\vartheta$ as below, the modification $\vartheta \circ M$

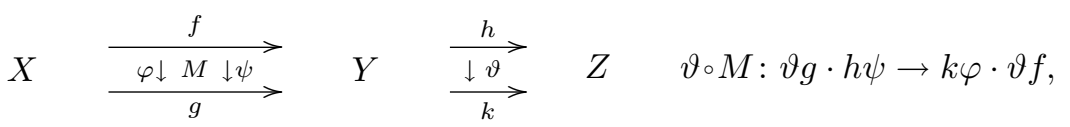


has a general cell $(\vartheta \circ M) x$ given by the following pastings (which are equal by axiom (lnt.3), applied to the lax natural transformation $\vartheta$ and the cell $M x$ )
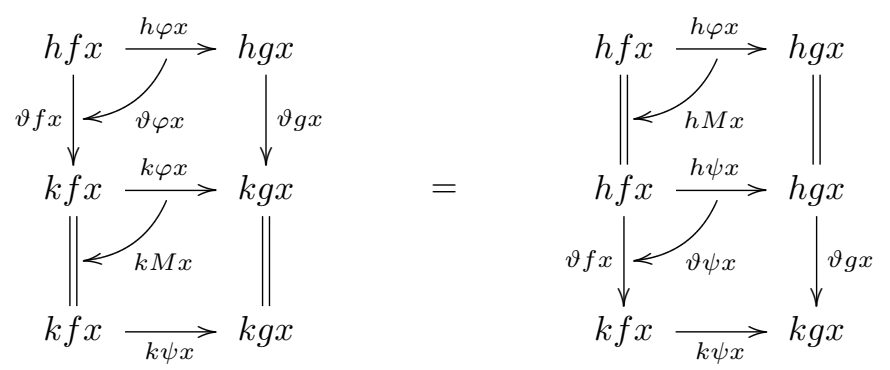

(b) Second, in the 'symmetric' situation, the general cell $(M \circ \vartheta) z$ of the modification $M \circ \vartheta$

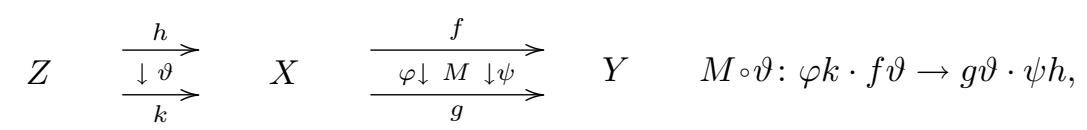

is each of the following pastings:

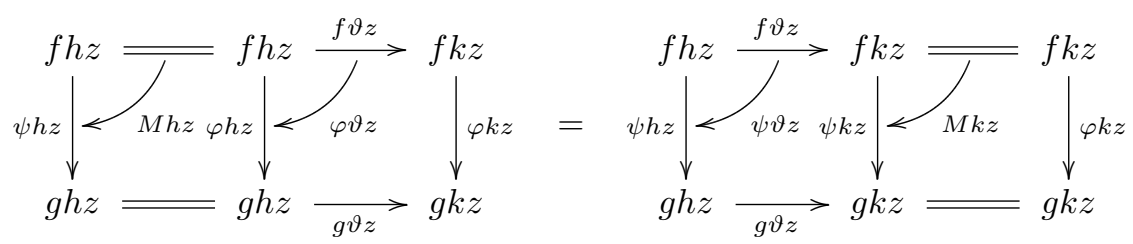

which are equal by axiom (mdf), for the modification $M$ and the map $\vartheta z: h z \rightarrow k z$.

\section{References}

[1] A. Bauer, L. Birkedal, D.S. Scott, Equilogical spaces, Theoret. Comput. Sci., 315 (2004), 35-59.

[2] R. Betti, S. Kasangian, A quasi-universal realization of automata, Rend. Istit. Mat. Univ. Trieste, 14 (1982), 41-48.

[3] R. Betti, A.J. Power, On local adjointness of distributive bicategories, Boll. Un. Mat. Ital. B, 2 (1988), 931-947.

[4] M.C. Bunge, Coherent extensions and relational algebras, Trans. Am. Math. Soc., 197 (1974), 355-390.

[5] L. Fajstrup, E. Goubault, M. Raussen, Algebraic topology and concurrency, preprint, 1999.

[6] L. Fajstrup, M. Raussen, E. Goubault, E. Haucourt, Components of the fundamental category, Appl. Categ. Struct., 12 (2004), 81-108. 
[7] P. Gaucher, A model category for the homotopy theory of concurrency, Homology, Homotopy Appl., 5 (2003), 549-599.

[8] P. Gaucher, E. Goubault, Topological deformation of higher dimensional automata, preprint, 2001. http://arXiv.org/abs/math.AT/0107060.

[9] E. Goubault, Geometry and concurrency: a user's guide, in: Geometry and concurrency, Math. Struct. Comput. Sci. 10(4) (2000), 411-425.

[10] M. Grandis, Cubical monads and their symmetries, in: Proceedings of the Eleventh International Conference on Topology, Trieste 1993, Rend. Ist. Mat. Univ. Trieste 25 (1993), 223-262.

[11] M. Grandis, Directed homotopy theory, I. The fundamental category, Cah. Topol. Géom. Différ. Catég. 44 (2003), 281-316.

[12] M. Grandis, Higher fundamental groupoids for spaces, Topol. Appl. 129 (2003), 281-299.

[13] M. Grandis, Inequilogical spaces, directed homology and noncommutative geometry, Homology Homotopy Appl. 6 (2004), 413-437.

[14] M. Grandis, The shape of a category up to directed homotopy, Theory Appl. Categ. 15(4) (2005), 95-146.

[15] J.W. Gray, Formal category theory: adjointness for 2-categories, Lecture Notes in Mathematics, Vol. 391, Springer-Verlag, Berlin, 1974.

[16] C.B. Jay, Local adjunctions, J. Pure Appl. Algebra 53 (1988), 227-238.

[17] G.M. Kelly, On clubs and doctrines, in: Category Seminar, Sydney 1972-1973, Lecture Notes in Mathematics, Vol. 420, Springer, Berlin, 1974, pp. 281-375.

[18] D. Scott, A new category? Domains, spaces and equivalence relations, Unpublished manuscript, 1996. http://www.cs.cmu.edu/Groups/LTC/.

[19] R. Street, Categorical structures, in: Handbook of Algebra, Vol. 1, North Holland, Amsterdam, 1996, pp. 529-577.

Marco Grandis grandis@dima.unige.it

Dipartimento di Matematica

Università di Genova

Via Dodecaneso 35

16146-Genova

Italy

This article is available at http://intlpress.com/HHA/v8/n1/a2/ 